NBER WORKING PAPER SERIES

\title{
ADDICTION AND CUE-CONDITIONED COGNITIVE PROCESSES
}

\author{
B. Douglas Bernheim \\ Antonio Rangel \\ Working Paper 9329 \\ http://www.nber.org/papers/w9329 \\ NATIONAL BUREAU OF ECONOMIC RESEARCH \\ 1050 Massachusetts Avenue \\ Cambridge, MA 02138 \\ November 2002
}

We would like to thank George Akerlof, Gadi Barlevy, Michele Boldrin, Kim Border, Samuel Bowles, Colin Camerer, Luis Corchon, David Cutler, Alan Durell, Victor Fuchs, Ed Glaeser, Jonathan Gruber, Justine Hastings, Jim Hines, Matthew Jackson, Chad Jones, Patrick Kehoe, Narayana Kocherlakota, Botond Koszegi, David Laibson, Darius Lakdawalla, Ricky Lam, John Ledyard, George Lowenstein, Ted O’ Donahue, David Pearce Christopher Phelan, Wolfgang Pesendorfer, Edward Prescott, Matthew Rabin, Paul Romer, Pablo Ruiz-Verdu, Andrew Samwick, Ilya Segal, Jonathan Skinner, Stephano de la Vigna, Bob Wilson, Leeat Yariv, Jeff Zwiebel, seminar participants at U.C. Berkeley, Caltech, Carlos III, Dartmouth, Harvard, Hoover Institution, Instituto the Analysis Economico, LSE, Michigan, NBER, Northwestern, UCSD, Yale, Wisconsin-Madison, SITE, Federal Reserve Bank of Minneapolis, and the McArthur Preferences Network for useful comments and discussions, and Luis Rayo for outstanding research assistance. Antonio Rangel gratefully acknowledges financial support from the NSF (SES-0134618), and thanks the Hoover Institution for its financial support and stimulating research environment. This paper was prepared in part while Bernhiem was a Fellow at the Center for Advanced Study in the Behavioral Sciences (CASBS), where he was supported in part by funds from the William and Flora Hewlett Foundation (Grant \#2000-5633). The views expressed herein are those of the author and not necessarily those of the National Bureau of Economic Research.

(C) 2002 by B. Douglas Bernheim and Antonio Rangel. All rights reserved. Short sections of text, not to exceed two paragraphs, may be quoted without explicit permission provided that full credit, including (C) notice, is given to the source. 
Addiction and Cue-Conditioned Cognitive Processes

B. Douglas Bernheim

Antonio Rangel

NBER Working Paper No. 9329

November 2002

JEL No.D0, D1, D6, D9, H0, H2, H5, I0, I1, K1, K4

\section{ABSTRACT}

We propose an economic theory of addiction based on the premise that cognitive mechanisms such as attention affect behavior independently of preferences. We argue that the theory is consistent with foundational evidence (e.g. from neuroscience and psychology) concerning the nature of decision-making and addiction. The model is analytically tractable, and it accounts for a broad range of stylized facts concerning addiction. It also generates a plausible qualitative mapping from the characteristics of substances into consumption patterns, thereby providing a basis for empirical tests. Finally, the theory provides a clear standard for evaluating social welfare, and it has a number of striking policy implications.

B. Douglas Bernheim

Department of Economics

Stanford University

Stanford, CA 94305-6072

and NBER

bernheim@stanford.edu
Antonio Rangel

Department of Economics

Stanford University

Stanford, CA 94305-6072

and NBER

rangel@leland.stanford.edu 


\section{Introduction}

Each year, millions of U.S. consumers spend hundreds of billions of dollars on addictive substances. ${ }^{1}$ Estimates for 1999 place total expenditures on tobacco products, alcoholic beverages, cocaine, heroin, marijuana, and methamphetamines at more than $\$ 150$ billion. During a single month in 1999, more than 57 million individuals smoked at least one cigarette, more than 41 million engaged in binge drinking (involving five or more drinks on one occasion), and roughly 12 million used marijuana. In 1998, slightly more than 5 million Americans qualified as "hard-core" chronic drug users. Roughly 4.6 million persons in the workforce met the criterion for a diagnosis of drug dependence and 24.5 million had a history of clinical alcohol dependence. In 1998, additional social costs resulting from health care expenditures, loss of life, impaired productivity, motor vehicle accidents, crime, law enforcement, and welfare totalled $\$ 185$ billion for alcohol and $\$ 143$ billion for other addictive substances. Smoking killed roughly 418,000 people in 1990, alcohol accounted for 107,400 deaths in 1992, and drug use resulted in 19,277 deaths during 1998. Alcohol abuse contributed to 25 to 30 percent of violent crimes. In 1999, more than 625,000 individuals were incarcerated for drug-related offenses.

Public policies regarding addictive substances run the gamut from laissez faire to taxation, subsidization (e.g. of rehabilitation programs), regulated dispensation, criminalization, product liability, and public health campaigns. Each alternative policy approach has passionate advocates and detractors. Economic analysis can potentially inform this debate, but it requires the analyst to adopt a theory of addiction.

The ideal economic theory of addiction would satisfy four criteria. First, it would be consistent with foundational evidence (e.g. from neuroscience and psychology) concerning the nature of decision-making and addiction. Second, it would account for the salient aspects of addictive behavior. Third, it would lend itself to tractable mathematical modeling. Fourth, it would provide a clear standard for policy evaluation (i.e. the measurement of social welfare).

A number of authors (such as Becker and Murphy [1988], Laibson [2001], Hung [2000], and Orphanides and Zervos [1995]) have proposed theories of addiction based on standard economic models of decision making. Others (such as Gul and Pesendorfer [2001b], Laibson [2001], and Gruber and Koszegi [2001]) have explored various "behavioral" alternatives. Unfortunately, as we discuss in we section 2, each of these theories falls short of the ideal. Among other shortcomings, each fails to explain important aspects of addictive behavior.

\footnotetext{
${ }^{1}$ The statistics in this paragraph were obtained from the following sources: Office of National Drug Control Policy [2001a,b], U.S. Census Bureau [2001], Gerstein et. al. [1999], National Institute on Drug Abuse [1998], National Institute on Alcohol Abuse and Alcoholism [2001], and Center for Disease Control [1993]. There is, of course, disagreement as to many of the reported figures.
} 
The purpose of this paper is develop an alternative theory of addiction that satisfies the four criteria articulated above. As our starting point, we depart fundamentally from standard economic theory by accepting the validity of the hypothesis that cognitive mechanisms such as attention, which determine how an individual thinks about a decision, affect behavior entirely apart from preferences. For example, an individual may fail to choose an alternative simply because he does not consider it, or because he fails to consider particular consequences. When this occurs, the individual may choose something other than the alternative that he would most prefer if he considered all options and consequences. Since this mistake results from an improper characterization of the decision problem, we refer to the phenomenon as characterization failure.

Our theory proceeds from the premise that environmental cues affect the way the brain characterizes decision problems. In particular, with experience, the brain develops cognitive shortcuts involving functions such as attention. These shortcuts appear to be mediated by, or at least associated with internal visceral states. For example, when someone notices the familiar smells of a barbecue, he experiences visceral sensations of hunger, and his thoughts turn to the acquisition and consumption of food. The use of a cognitive shortcut does not necessarily lead to characterization failure; on the contrary, an efficient shortcut could economize on the costs of decision making by focusing attention on the most promising alternatives and pertinent consequences. Nevertheless, in the context of addictive substances, the evidence suggests that cognitive shortcuts focus attention on inappropriate actions and consequences given the individual's objectives and preferences. Thus, environmental cues associated with past usage influence current use through cognition (e.g. which alternatives and consequences capture the brain's attention) as well as through preferences.

We provide a parsimonious representation of this phenomenon in an otherwise standard model of rational addiction. Specifically, we allow for the possibility that the individual may enter a "hot" cognitive mode in which he always chooses to consume the substance irrespective of underlying preferences (implicitly because inappropriate cognitive shortcuts focus attention on usage and the associated "high"), and we assume that the likelihood of entering this state is related to past choices (implicitly because, through conditioning, previous usage increases the probability of encountering environmental cues which trigger the hot cognitive mode). The individual may also operate in a "cold" cognitive mode, wherein he considers all alternatives and contemplates all consequences, including the effects of current choices on the likelihood of entering the hot cognitive mode in the future. ${ }^{2}$

\footnotetext{
${ }^{2}$ Our analysis is most closely related to work by Loewenstein [1996,1999], who considers simple models in which an individual can operate in either a hot or cold decision-making mode. Lowenstein's approach differs from ours in at least one important respect: he assumes that behavior in the hot mode reflects the application of a "false" utility function, rather than a particular (and potentially flawed)
} 
As a matter of formal mathematics, our model involves a minimalistic departure from the standard framework. Behavior corresponds to the solution of a dynamic programming problem with stochastic state-dependent mistakes. Our approach therefore harmonizes economic theory with foundational evidence on decision making and addiction without sacrificing analytic tractability.

The model has several attractive features. It explains a broad range of important stylized facts associated with addiction. It generates a plausible qualitative mapping from the characteristics of substances into consumption patterns, thereby providing the basis for empirical tests. It gives rise to a clear welfare criterion, and it has some surprising public policy implications. For example, in some circumstances it is optimal to subsidize the use of addictive substances even though consumption is excessive. Yet under the same circumstances, criminalization may be superior to taxation.

The remainder of the paper is organized as follows. Section 2 describes patterns of addictive behavior. It also briefly summarizes and evaluates existing theories of addiction. Section 3 discusses foundational evidence concerning the nature of decisionmaking and addiction. Section 4 presents our formal model. Section 5 explores the model's positive implications, including its ability to generate observed consumption patterns and to explain the main stylized facts concerning addiction. Section 6 examines the welfare implications of various public policies. Section 7 concludes. Proofs of propositions appear in an appendix.

\section{Addictive Behavior}

\subsection{Patterns of addictive behavior}

The consumption of addictive substances has received substantial attention in neuroscience, psychology, epidemiology, sociology, and economics. ${ }^{3}$ From this extensive body of research, we have distilled eight stylized facts which, we argue, should serve as a litmus test for evaluating the validity of any economic theory of addiction.

First, short-term abstention is common even for the most addictive substances, but long-term recidivism rates are high (see Goldstein [2001], Hser, Anglin, and Powers [1993], Harris [1993], and O'Brien [1997]). In many instances, addicts attempt to "kick the habit," but are ultimately unsuccessful. For example, during 2000, 70 percent of current smokers expressed a desire to quit completely and 41 percent stopped smoking

mode of cognition. See also Loewenstein and Lerner [2001] for an excellent review of the evidence concerning the effects of emotions and visceral states on decision-making.

${ }^{3}$ Gardner and David [1999] provide the following list of addictive substances: (1) alcohol, (2) barbiturates, (3) amphetamines, (4) cocaine, (5) caffeine and related methylxanthine stimulants, (6) cannabis, (7) hallucinogenies, (8) nicotine, (9) opioids, (10) dissociative anasthetics, and (11) volatile solvents. 
for at least one day in an attempt to quit, but only 4.7 percent successfully abstained for more than three months. ${ }^{4}$

Second, consumption and recidivism are associated with cue-conditioned cravings. Recidivism rates are especially high when addicts are exposed to cues related to their past drug consumption (Goldstein [2001], Goldstein and Kalant [1990], O'Brien [1976,1997], Robins [1974], Robins et. al. [1974], and Hser et. al.). Long-term usage is considerably lower among those who experience significant changes of environment. ${ }^{5}$ For this reason, drug treatment programs advise recovering addicts to move to new locations, or at least to avoid the places where previous consumption took place. A recovering addict is also significantly more likely to "fall off the wagon" if he receives a small taste of his drug-of-choice (Goldstein [2001]). This phenomenon, known as "priming," suggests that even minimal exposure to the substance serves as a powerful cue that activates cravings.

Third, addicts continue to use drugs compulsively even though with sufficiently sustained use they develop tolerance with respect to the hedonic effects of the substance (i.e., the quality and intensity of the high often diminishes despite increases in dosage). The development of hedonic tolerance is a complex process. ${ }^{6}$ For some drugs, such as cocaine, users experience a phenomenon called sensitization, in which the hedonic effects of the drug are enhanced in the short term, for example during binges. Nevertheless, there is some agreement that a large fraction of substances and users develop hedonic tolerance with sustained use. In a recent review of the neurobiology of addiction, Hyman and Malenka [2001,p. 695] observe:

"The desire to elevate or otherwise alter mood often motivates initial drug use. However, the pleasure (or relief of dysphoric moods) produced by drugs often habituates; for drugs such as alcohol and nicotine, pleasure can be markedly reduced over time by medical complications. Addictive individuals sometimes describe their continuing drug use as an attempt to re-experience remembered 'highs' often without success."

Similarly, Goldstein [2001, p. 86] states that "with most addictive drugs, repeated

\footnotetext{
${ }^{4}$ Notably, more educated individuals were far more likely to quit successfully, even though education bore little relation either to the desire to quit or to the frequency with which smokers attempted to quit (Trosclair et. al. [2002]).

${ }^{5}$ Robins [1974] and Robins et.al. [1974] found that Vietnam veterans who were addicted to heroin and/or opium at the end of the war experienced much lower relapse rates than other young male addicts during the same period. A plausible explanation is that veterans encountered fewer environmental triggers (familiar circumstances associated with drug use) upon returning to the U.S.

${ }^{6}$ The term tolerance is used to describe a wide range of physical adaptations that take place in response to the addictive substances. For example, with repeated usage, the body (in particular the liver) develops an increased capacity to destroy the drug. This leads to a phenomenon called metabolic tolerance (see Goldstein [2001]).
} 
administration over a long time ... leads to a loss of effect, so that more and more is needed to produce the same high as before." A user-oriented website concurs: ${ }^{7}$ "Tolerance builds up rapidly after a few doses and disappears rapidly after a couple of days of abstinence. Heavy users need as much as eight times higher doses to achieve the same psychoactive effects as regular users using smaller amounts. They still get stoned but not as powerfully."

Fourth, addicts often describe themselves as power less to regulate their consumption of the substance. They perceive some of their past choices as mistakes, in the sense that they think they would have been better off in the past as well as the present had they acted differently, even when no learning has occurred. They sometimes characterize current choices as mistakes even in the act of consumption. ${ }^{8}$ They also recognize that they are likely to make similar mistakes in the future. It is instructive that the twelve-step program of Alcoholic Anony mous begins as follows: "We admitted we were powerless over alcohol - that our lives had become unmanageable." 9

Fifth, users respond to standard economic incentives such as prices and information about the effects of addictive substances. ${ }^{10}$ For example, an aggressive U.S. public health campaign is widely credited with reductions in rates of cigarette smoking. There is also evidence that users engage in sophisticated forward-looking deliberation, reducing current consumption in response to future price increases (Gruber and Koszegi [2001]).

Sixth, addicts attempt to control use through various pre-commitments, such as checking into rehabilitation centers and consuming medications that either generate unpleasant side effects or reduce pleasurable sensations if the substance is subsequently consumed. Disulfiram interferes with the liver's ability to metabolize alcohol; as a result, ingestion of alcohol produces a highly unpleasant physical reaction for a period of time. Methadone, an agonist, activates the same opioid receptors as heroin, and thus produces a mild high, but has a slow-onset and a long-lasting effect. It thereby reduces the high produced by heroin. Naltrexone, an antagonist, blocks specific brain receptors, and thereby diminishes the high produced by opioids. All of these treatments reduce the frequency of relapse. ${ }^{11}$

Seventh, use is sensitive to the deployment of attention. Exogenous attention shocks can temporarily discourage use without providing new information. A recover-

\footnotetext{
${ }^{7}$ See htpp://www.thegooddrugsguide.com/cannabis/addiction.htm.

${ }^{8}$ Goldstein [2001,p.249] describes this phenomenon as follows: the addict had been "suddenly overwhelmed by an irresistible craving, and he had rushed out of his house to find some heroin. ... it was as though he were driven by some external force he was powerless to resist, even though he knew while it was happening that it was a disastrous course of action for him" (italics added).

${ }^{9}$ See http://www.alcoholics-anonymous.org/english/E_FactFile/M-24_d6.html.

${ }^{10}$ See Chaloupka and Warner [2001] and MacCoun and Reuter [2001] for a review of the evidence.

${ }^{11}$ See O’Brien [1997] and Goldstein [2001].
} 
ing addict is, for example, less likely to use (at least temporarily) if, while experiencing a strong craving, he is reminded of undesirable consequences with which he is already familiar. ${ }^{12}$ Consequently, recovering addicts exhibit a demand for attention management therapies. Even addicts who have stayed clean for years attend support group meetings, such as Alcoholics Anonymous, which provide no new information. ${ }^{13}$

Eighth, patterns of usage vary dramatically across addictive substances and, for any given substance, across methods of administration and users. Caffeine is consumed on a regular basis and users rarely seek clinical intervention to control use, while cocaine users experience binging cycles and sometimes seek institutional rehabilitation. Cocaine and crack, though chemically identical, give rise to different consumption patterns. ${ }^{14}$ Although a sizable fraction of the population either experiments with drugs or uses for recreation, most do not become clinically addicted. ${ }^{15}$

\subsection{Existing theories}

Existing economic theories of addiction include (1) variations on the standard model of rational economic decision making (Becker and Murphy [1988]), including generalizations that allow for random shocks and state-contingent utility (Laibson [2001] and Hung [2000]), (2) models of "temptation" wherein well-being depends not only upon the chosen action but also on actions not chosen (Gul and Psendorfer [2001a,2001b] and Laibson [2001]), (3) models with present-biased preferences with either naive or sophisticated expectations (O’Donoghue and Rabin [1999,2000] and Gruber and Koszegi [2001]), and (4) models with "projection bias," wherein agents mistakenly assume that future preferences will resemble current preferences (Loewenstein [1996,1999], and Loewenstein, O'Donoghue, and Rabin [2001]). Each of these theories contributes to an understanding of decision-making in general and addiction in particular. Although a comprehensive discussion of the various behavioral alternatives is beyond the scope of the current paper, it is important to highlight some limitations of these approaches. For a more complete discussion, see Bernheim and Rangel [2002].

\footnotetext{
${ }^{12}$ There are, for example, references to the role of attention shocks in Massing [2000], who provides detailed descriptions of addicts' experiences.

${ }^{13}$ Goldstein [2001] reports that there is a shared impression among the professional community that 12-step programs such as AA (p. 149) "are effective for many (if not most) alcohol addicts." However, given the nature of these programs, objective performance tests are not available. The AA treatment philosophy is based on "keeping it simple by putting the focus on not drinking, on attending meetings, and on reaching out to other alcoholics." Goldstein also notes that, according to AA, there are recovering alcoholics, but not ex-alcoholics; hence the dictum "once an addict, always an addict."

${ }^{14}$ Crack is prepared from cocaine by mixing it with baking soda and water, and then boiling it. This has two important consequences. First, crack can be smoked, which allows the brain to absorb the substance more efficiently, and leads to a quicker and more intense high. Second, crack is significantly cheaper, which leads to a pattern of more frequent administration.

${ }^{15}$ See Goldstein and Kalant [1990], Gazzaniga [1990,1994], and Koob and Moal [1997].
} 
With respect to the first stylized fact, all of the preceding theories can, under appropriate assumptions, account for cycles of use and abstention, as well as for quitting by some users. If, however, one interprets an intention to quit "completely" as referring to all future contingencies, then recidivism among intended quitters involves a failure to follow through on a contingent plan. ${ }^{16}$ Such failures can occur with present-biased preferences if expectations are naive, or with projection bias, but they are inconsistent with the other possibilities mentioned above.

All of the existing theories are at odds with the third stylized fact, since they assume that the addictive substances are distinguished by intertemporal complementarities in consumption: the marginal utility of using the substance is assumed to increase with previous consumption. In fact, without intertemporal complementarities nothing would distinguish addictive and non-addictive substances; the very same self-control problem would influence the consumption of all immediately pleasurable activities, from injecting heroin to drinking water.

With respect to the fourth stylized fact, none of the existing theories can account for the observation that addicts sometimes describe their current choices as mistakes. In each instance, the decision maker maximizes a utility function that describes his well-being at the time of choice. The anticipation of future mistakes is inconsistent with the standard model, temptation preferences, hyperbolic discounting with naive expectations, and projection bias. In each of these cases individuals believe that their future behavior will be optimal when evaluated by current preferences. ${ }^{17}$ With presentbiased preferences and sophisticated expectations, the individual anticipates that future choices may be contrary to his current desires; however, he recognizes that those choices will be optimal for his in the future, and hence will not be mistakes when he makes them. Finally, all of these models are unable to account for the perception that past choices were mistakes. In every case, an individual might regret a past choice in the sense that he would be better off today had he acted differently, but he never believes that an alternative choice would have made him better off in the past as well as the present.

With respect to the sixth stylized fact, the standard framework is capable of explaining voluntary admission to rehabilitation clinics and related behaviors provided that these activities reduce the likelihood of experiencing cravings. But this is contrary to the experience of many addicts who check into rehabilitation centers not because they expect to avoid cravings, but rather precisely because they anticipate cravings and

\footnotetext{
${ }^{16}$ According to Hyman and Malenka [2001,p.697], there is agreement that "cue-initiated relapses can occur in individuals who have strongly resolved never to use drugs again, often without the addicted person having insight into what is happening to them" (italics added).

${ }^{17}$ Although this belief is false in the last two cases, individuals nonetheless fail to anticipate future mistakes.
} 
wish to control their reactions. Furthermore, even in instances where entering a rehabilitation center does reduce the likelihood of cravings (e.g. by removing environmental cues), the standard framework implies counterfactually that the addict would find the center's program more attractive if it made the substance available upon request (in case of cravings). Likewise, the standard framework is hard-pressed to explain the voluntary use of substances such as disulfiram, which simply reduce the utility derived from future usage. More generally, within the standard framework, the decision-maker would avoid precommitments (decisions that eliminate future options). Similar comments apply for models with projection bias, and with present-biased preferences and naive expectations. Models with temptation preferences can explain precommitments, but only if the elimination of the tempting alternative suppresses cravings. For the reasons described above, this explanation is problematic. Among the existing alternatives, only present-biased preferences with sophisticated expectations can account adequately for the fifth stylized fact.

All of the theories mentioned above also struggle to account for the seventh stylized fact. Since behavior is, in each instance, a direct manifestation of preferences at each moment in time, attention shocks cannot affect behavior. ${ }^{18}$

The second, fifth, and eighth stylized facts pose fewer problems for existing theories. Even though the literature contains many models of addiction that do not specifically encompass cue-conditioned cravings, an appropriately articulated version of each existing theory can nevertheless account for the second stylized fact (see, for example, Laibson's [2001] extension of Becker and Murphy's [1988] model). With intertemporal complementarities, these theories are also consistent with evidence indicating that usage is sensitive to both current and future economic incentives (the fifth stylized fact). While there has been no systematic attempt to account for the heterogeneity of consumption patterns across addictive substances, methods of administration, and users (the eighth stylized fact), each theory provides many dimensions of flexibility.

Additional reservations concerning existing theories of addiction include the following. First, some of the existing theories lack explicit neuro foundations. They are not intended to depict actual decision-making processes; rather, they are strictly "as if" representations of behavior. Second, some of the alternatives sacrifice mathematical tractability (relative to the standard model). Models with present-biased preferences introduce strategic considerations, and require one to depict behavior as the equilibrium of a game played between the decision-maker and his future incarnations. These equilibria can be extremely complex and challenging to characterize.

\footnotetext{
${ }^{18}$ This is not to say that the concept of attention is in itself problemmatic. As discussed in section 3 , even the standard framework can accomodate the notion that attention and preferences shift together in response to environmental cues. It is more challenging, however, to account for the observation that individuals learn to manage behavior by managing attention.
} 
It can also be difficult to analyze models of temptation when preferences are defined over sets rather than over choices. ${ }^{19}$ Third, with either present-biased preferences or temptation preferences, welfare is often ambiguous and a matter of the perspective chosen for evaluation.

\section{Foundations}

Our objective in this paper is to explain addictive behavior based on general principles concerning decision making, rather than as an idiosyncratic special case. The general principles that we invoke allow for the possibility that the cognitive portions of the brain's decision-making algorithms perform poorly in identifiable circumstances. We argue that addiction is a particularly severe instance of this phenomenon. In this section, we discuss the available evidence concerning these foundational hypotheses.

\subsection{Cue-Conditioned Characterization Failure}

Decision making involves (at least) three types of processes: characterization, evaluation, and hedonic experience. Characterization entails the deployment of cognitive mechanisms such as attention, memory, and forecasting to identify the state of the world, the set of possible actions, and the present and future consequences of each action. Evaluation refers to the process by which the brain assesses the desirability of each action under consideration in light of its projected consequences and the state of the world. Finally, a hedonic experience, consisting of both pleasant and unpleasant sensations, results from the state of the world and the consequences of choices.

Under appropriate assumptions concerning these three processes, one obtains the standard model of economic decision-making. For example, one could assume that the brain completely and correctly extrapolates the consequences of all possible alternatives during the characterization stage, and selects among these alternatives using a criterion that corresponds to maximization of discounted expected hedonic experience during the evaluation stage. In this paper we investigate the implications of more realistic assumptions concerning the nature of characterization. We note that there may also be valid reasons to depart from standard assumptions concerning evaluation and experience, but we do not pursue these possibilities here (see Bernheim and Rangel [2002] for a discussion of evidence regarding the other two processes).

Since the brain is a finite computational mechanism, it cannot consider every possible option and forecast every potential consequence. Characterization shortcuts are thus unavoidable. Given the current state of knowledge, it is not yet possible to describe

\footnotetext{
${ }^{19}$ Gul and Pesendorfer [2001a] demonstrate that one can depict choice with temptation as the solution to a fairly standard dynamic program under appropriately strong assumptions.
} 
cognitive decision-making algorithms with precision. Nevertheless, recent research findings in psychology and neuroscience provide foundations for three general principles. First, emotional and visceral states (e.g. anger or cravings) affect cognitive activities, including attention, that play a central role in characterization. Second, certain environmental cues systematically trigger particular visceral states and the associated cognitive modes. Third, these triggers are established through a form of learning called cue-conditioning: with experience the brain learns to associate particular cues with the visceral states that guide cognition to hedonically salient states, options and consequences.

We illustrate these three principles through a simple example. When an individual is hungry (a visceral state), his attention focuses on tasks associated with obtaining food. Specific environmental cues, such as the smell of a barbecue, can trigger sensations of hunger. Far from being hard-wired, this response is cue-conditioned: the aroma triggers hunger because the individual has had the pleasurable experience of consuming food at previous barbecues.

The use of cue-conditioned cognitive shortcuts does not by itself overturn the standard model. If, for example, visceral and emotional signals guide attention to appropriate subsets of alternatives and consequences, the brain may select an optimal or nearly optimal alternative even though it characterizes only a small portion of the decision problem. In fact, the use of such shortcuts could be an effective evolutionary adaptation. The problem, as emphasized by evolutionary psychologists (see Barkow, Cosmides, and Tooby [1995]), is that brain processes evolved to promote fitness in the hunter-gatherer world, not in the modern world. Thus, for example, panic-triggered flight responses that helped humans escape from predators as hunter-gatherers may be counterproductive when judged by the individual's own objectives and preferences in many modern situations.

Accordingly, we depart from the standard model by assuming that, in some circumstances, environmental cues can induce visceral states that divert attention from the most preferred alternatives and/or hedonically salient consequences. When this happens, the ability of the brain to choose the most preferred option is impaired. We refer to this phenomenon as cue-conditioned characterization failure.

If an individual repeatedly experiences characterization failure upon encountering particular environmental conditions, he may learn to associate those conditions with poor decision-making. This type of self-understanding may lead to a range of interesting and economically important behavioral patterns. For example, individuals may avoid situations in which they are exposed to certain cues (cue avoidance), attempt to preclude alternatives that they tend to choose when experiencing characterization failure (precommitment), desensitize themselves to problematic cues, or develop self- 
management techniques to counteract the effects of strong visceral states (such as counting to ten before acting).

Research in several disciplines provides foundational evidence for various aspects of the hypotheses described above. First, visceral states appear to influence the outcome of decision-making even when they are arguably uncorrelated with pertinent aspects of preferences. Shoppers tend to purchase more food at the grocery store when they are hungry, even though they know that the state of hunger is temporary (see Abratt and Goodey [1990]). A variety of tactics used in the contexts of interrogations and legal depositions are intended to elicit responses with long-lasting implications by inducing transitory emotional reactions (Loewenstein [1996]). Likewise, salespeople often attempt to influence consumers' choices by manipulating visceral desires through environmental cues, even when the good in question is durable while the visceral state is not.

Second, a series of experiments by Mischel and coauthors suggest that self-control is sensitive to the deployment of attention and to the activation or non-activation of particular thoughts. ${ }^{20}$ A subject (typically a child) is placed in a room and is offered a choice between an inferior prize and a superior one (one or two pieces of candy). Subjects can obtain the inferior prize at any time by calling the experimenter, but must wait until the experimenter returns to obtain the superior prize. In practice, the child's ability to wait depends crucially on whether the inferior prize is visible. Merely covering the object significantly enhances patience.

More generally, in Mischel's experiments, the deployment of attention emerges as a key determinant of self-control. Any stimulus that focuses attention on the "tempting" features of the inferior prize increases the likelihood that the child will select it. Children are significantly more likely to wait if they are advised to distract themselves by thinking about something else, or if they are provided with a toy, even when children in a control group show no interest in the toy. Advising children not to think about the prize is counterproductive, since this induces them to repeatedly check whether or not they are thinking about it, thereby inadvertently activating thoughts about the prize. $^{21}$ During the course of development, children acquire self-understanding, and begin to consciously regulate thought-generating environmental cues ("metacognitive awareness"). ${ }^{22}$ When asked whether they would prefer to have the prize exposed or covered, children under four exhibit no preference and are unable to justify their choice. In contrast, those over five prefer to wait with the prize hidden, and offer explanations that suggest some understanding of the principle that exposure to the prize influences

\footnotetext{
${ }^{20}$ See Mischel [1974], Mischel and Moore [1973], Mischel, Shoda, and Rodriguez [1992], and Metcalfe and Mischel [1999].

${ }^{21}$ This variation of the experiments is closely related to the work of Wegner [1994].

${ }^{22}$ See Metcalfe and Mischel [1999].
} 
attention. These findings are consistent with the hypothesis that seeing or thinking about the prize triggers strong visceral states (cravings) that restrict the child's subsequent thoughts to a limited range of activities and outcomes. ${ }^{23}$

In some settings, there is also direct evidence that visceral states influence behavior by restricting attention to limited sets of alternatives and consequences. In particular, fear focuses attention on the possibility of environmental threats (Janis [1967]) and on a limited number of "fight-or-flight" responses (Panksepp [1988], ch. 11).

Third, research in neuroscience has identified some of the mechanisms through which visceral states influence choice by altering cognition. LeDoux's work on fear is a leading example. ${ }^{24}$ Information about the environment reaches the amygdala (a primitive brain structure that helps to initiate responses to sensory stimuli) via two principal routes: a short "direct" route, and a long cortical route. Along the first route, information passes directly from the sensory thalamus to the amygdala without intermediate processing by the neocortex. Along the second route, information is sent from the sensory thalamus to various neocortical structures, where it is processed before proceeding to the amygdala. The short route is more primitive (in an evolutionary sense) and permits the organism to initiate rapid responses in critical survival situations. Though slower, use of the long route permits more deliberate responses. The existence of the short route implies that, in some circumstances, human behavior can result with little (if any) cognitive deliberation. ${ }^{25}$

Finally, research in neuroscience also suggests that individuals cannot make sound decisions unless visceral states guide cognition. This principle finds support in a series of influential neurological studies by Antonio Damasio and various coauthors concerning the decision-making abilities of patients with damage to the ventromedial sector of the prefrontal cortex. ${ }^{26}$ Injuries of this variety lead to abnormal (often muted) emotional responses, even though a standard battery of tests reveals no cognitive impairments. Although their "logical" reasoning facilities are intact, these individuals nevertheless exhibit an impaired capacity for sound decision making. Based on these findings, Damasio has formulated the "somatic marker hypothesis," which holds that, in normal individuals, the brain uses visceral states to simplify complex decision problems. In Damasio's theory, the ventromedial frontal cortex contains dispositional information

\footnotetext{
${ }^{23}$ Metcalfe and Mischel [1999] reach similar conclusions.

${ }^{24}$ See LeDoux $[1992,1993,1998]$ and also Davis [1992a,1992b].

${ }^{25}$ Consider the following example (LeDoux, [1998]). While hiking through a park, an individual glimpses a long stick, resembling a snake, lying on the ground. This information first reaches his amygdala through the short route. The amygdala automatically initiates defensive responses, including autonomic changes such as increased blood circulation, endocrine changes such as the release of adrenaline, and neocortical changes such as heightened alertness. Before consciously thinking about alternatives, the hiker stops short or leaps to safety.

${ }^{26}$ See Damasio [1994], Behara et al. [1996,1997], and Bechara et. al. [1994].
} 
which is accumulated through experience. In any given visceral state, pre-conscious processing uses this information to identify appropriately "marked" alternatives, which then receive conscious consideration.

\subsection{Addictive Substances and Cue-conditioned Characteriza- tion Failure}

For several decades neurobiologists have recognized that a variety of addictive substances, from alcohol to cocaine, have a powerful impact on the brain's mesolimbic dopamine system (MDS) (see, for example, Hyman and Malenka [2001], Nestler [2001], and Wickelgreen [1997] for recent reviews). ${ }^{27}$ The MDS, in turn, plays a central role in the regulation of basic behaviors. For example, experiments have shown that rats who are given drugs that block dopamine receptors, thereby impeding the appropriate operation of the MDS, eventually stop feeding (Berridge [1999]).

Experiments have also shown that direct stimulation of the MDS is a powerful way to induce experimental subjects to perform a behavior. For example, in a series of classic experiments, Olds and Milner [1954] demonstrated that rats learn to return to locations where they have received direct electrical stimulation to the MDS. When provided with opportunities to self-administer by pressing a lever, the rats rapidly became addicted, giving themselves approximately 5,000-10,000 "hits" during each one hour daily session, ignoring food, water, and opportunities to mate. Addicted rats were willing to endure painful electric shocks to reach the lever. ${ }^{28}$ Similarly, when rats are allowed to self-administer cocaine, they ignore hunger, reproductive urges, and all other drives, consuming the substance until they die (Pickens and Harris [1968] and Gardner and David [1999]).

Based on these findings, researchers proposed a variety of theories that linked the consumption of addictive substances to the their ability to generate enormous hedonic rewards (the "high") by stimulating the MDS. This view parallels existing economic theories in which users consume addictive substances to maximize pleasure.

Neurobiological support for this "pleasure principle" theory of addiction has eroded over the course of the last decade with the accumulation of new evidence indicating that MDS activity does not exclusively, and perhaps not even primarily, relate to the

\footnotetext{
${ }^{27} \mathrm{Of}$ the addictive substances listed in a previous footnote, only hallucinogenics (or psychedelics) do not seem to produce intense stimulation of the MDS. Instead, they act on a "subtype of serotonin receptor which is widely distributed in areas of the brain that process sensory inputs" (Goldstein $[2001, \mathrm{p} .231])$. There is some disagreement as to whether hallucinogens are properly classified as addictive substances (see Goldstein [2001, ch. 14]). Notably, laboratory animals and humans learn to self-administer the same set of substances, with the possible exception of hallucinogenics (Gardner and David [1999, p.97-98]).

${ }^{28}$ See Gardner and David [1999] for a summary of these experiments.
} 
generation of pleasure. ${ }^{29}$ This evidence includes the following findings. First, unpleasant and novel stimuli that are hedonically neutral also trigger a release of dopamine (Becerra et. al. [2001] and Schultz [1998, 2000]). Second, dopamine surges in anticipation to rewards, or cues associated with rewards, not during or after their consumption. Furthermore, dopamine cells respond to rewards only when they occur unexpectedly. This suggests that the MDS acts more as a learning mechanism than as a hedonic meter (see Schultz, Dayan, and Montague [1997], and Schultz [1998, 2000]). Third, using advanced imaging technology, Breiter et. al. [1997] have scanned addicts' brains during complete usage episodes, and found that the dopamine system remains active long after the "high" has passed. Fourth, rats have normal hedonic reactions to sweet and bitter tastes, and can learn about new hedonic stimuli, even when their ability to transmit dopamine has been impaired through the administration of a neurotoxin (Berridge and Robinson [1998]).

The accumulating evidence has lead neurobiologists to a new consensus view of addiction. In this view, addictive substances directly affect brain processes, such as memory and attention, that are central to deliberative decision-making. Moreoever, these effects are poorly correlated with hedonic pleasure (i.e., preferences). According to Hyman and Malenka's [2001, p. 703] review of the pertinent literature, "recent evidence ... (suggests) that the central behavioral features of addiction result from the ability of drugs to usurp normal mechanisms of memory in crucial survival circuits." 30 This may help to explain the observation that addicts' thoughts tend to focus almost exclusively on consumption of substances during binges and while experiencing cravings (Gawin [1991]). ${ }^{31}$ Notably, the literature draws a distinction between liking a substance, which results from the experience of hedonic pleasure, and wanting a substance, which refers to aspects of decision processes that incline an addict towards usage. It also emphasizes that wanting and liking are not always aligned. Robinson and Berridge [2000,p. 91] conclude that "the brain systems that are sensitized do not mediate the pleasurable or euphoric effects of drugs (drug 'liking'), but instead

\footnotetext{
${ }^{29}$ Wickelgreen [1997, p. 35] quotes Roy Wise, one of the individuals who originally proposed the pleasure principle theory of addiction, as follows: "I no longer believe that the amount of pleasure felt is proportional to the amount of dopamine floating around in the brain."

${ }^{30}$ Vorel et. al. [2001] have shown that the stimulation of memory centers can trigger strong cravings and recidivism among rats that have previously self-administered cocaine (Vorel and Gardner [2001] and Holden [2001a,b] provide non-technical discussions). Ungless et. al. [2001] have shown that similar cellular mechanisms may be at work in memory and addiction (see Helmuth [2001] for a non-technical discussion).

${ }^{31}$ Tiffany [1990, p. 152] summarizes various findings as follows: “Over a history of repeated practice, the cognitive systems controlling many aspects of drug procurement and consumption take on the character of automatic systems. Thus, drug-use behaviors tend to be relatively fast and efficient, readily enabled by particular stimulus configurations, initiated and completed without intention, difficult to impede in the presence of triggering stimuli, effortless, and enacted in the absence of awareness."
} 
they mediate a subcomponent of reward that we have termed incentive salience (drug 'wanting')."32

The preceding findings provide neurobiological foundations for modeling addicts as susceptible to cue-conditioned characterization failure. In our language, these findings suggest that addictive substances divert addicts from optimal choices (judged according to their own preferences, or "liking") by promoting the development of inappropriate decision-making shortcuts (i.e. the processes governing "wanting").

It is important to emphasize that the mechanisms involved in addiction are complex and not yet fully understood. Although there is ample evidence of a discrepancy between "drug liking" (preferences) and "drug wanting" (decision-processes) among addicts and experimental animals, a satisfactory explanation for the existence of this discrepancy does not yet exist. We conjecture that evolution callibrated the process of selecting decision-making shortcuts for problems resembling those encountered in nature, and that this process is not properly callibrated for substances, such as drugs, which activate the MDS with unnatural strength. Notably, most addictive substances are not found naturally in highly potent forms, and were therefore not encountered during the course of human evolution. Their ability to activate the MDS with great potency, and thereby influence the shortcut-creation process, is something of a biochemical fluke, rather than an evolutionary adaptation.

\section{The Model}

In this section, we present a tractable model of addiction that is consistent with the foundational principles discussed in section 3. The model is based on the central simplifying assumption that the decision maker (DM) operates in one of two cognitive modes (denoted by $\mu$ ): a cold mode $(\mu=C)$, in which the brain characterizes the decision problem perfectly, and a hot mode $(\mu=H)$, in which there is a extreme form of characterization failure. The DM lives for an infinite number of discrete periods. Within each period, he makes two choices (sequentially). First, he selects an activity, which we interpret as a "lifestyle" choice for the current period. Second, he decides whether to use an addictive substance or abstain. He always makes the first decision in the cold mode, but can make the second decision in either the cold or hot mode. The prevailing cognitive mode for the second decision depends upon environmental conditions, which are in turn influenced by the first decision. Once in the hot mode,

\footnotetext{
${ }^{32}$ Tiffany [1990, p.152] summarizes the pertinent research as follows: “Over a history of repeated practice, the cognitive systems controlling many aspects of drug procurement and consumption take on the character of automatic systems. Thus, drug-use behaviors tend to be relatively fast and efficient, readily enabled by particular stimulus configurations, initiated and completed without intention, difficult to impede in the presence of triggering stimuli, effortless, and enacted in the absence of awareness."
} 
he invariably attempts to use the substance even if his underlying preferences favor abstention. Current usage increases the likelihood of triggering the hot mode in subsequent periods. It can (but need not) also have an effect on the baseline level of well-being and on the pleasure derived from consumption in the future.

Formally, the DM enters each period in one of $S+1$ addictive states, labelled $s=0,1, \ldots, S$, which summarize the history of use. These addictive states evolve as follows. Usage in state $s \geq 1$ leads to state $\min \{S, s+1\}$ in the next period. No use leads to state $\max \{1, s-1\}$. Note that it is impossible to reach state 0 from any state $s \geq 1$. However, the reverse is not true. In state $s=0$, use leads to state 1 , while no use leads to state 0 . The usage state $s=0$ represents a "virgin state" in which the DM has had no contact with the substance. We use $y_{s}$ to denote the DM's single-period income when he is in state $s$.

At the beginning of each period, the DM chooses a "lifestyle" activity a from the set $\{E, A, R\}$. Activity $E$ ("exposure") entails a high likelihood that the DM will encounter environmental conditions that trigger the hot mode. Examples include attending parties at which the substance is readily available. Activity $A$ ("avoidance") is less intrinsically enjoyable than $E$, but entails a lower likelihood of exposure to environmental triggers. Examples include staying at home to read or attending AA meetings. Activity $R$ ("rehabilitation") entails a commitment to clinical treatment at a residential center during the current period. Activity $R$ is even less intrinsically enjoyable than $A$, it further reduces the likelihood of exposure to known environmental triggers, and it guarantees abstention during the current period because the substance becomes unavailable. It also entails a monetary $\operatorname{cost} r_{s} \geq 0$ (which may depend upon the DM's addictive state).

After the DM selects an activity, events outside of his control randomly generate environmental cues, which potentially influence his cognitive mode and decision processes for the duration of the period (recall that the DM always enters the next period in the cold mode). For any initial choice $a \in\{E, A, R\}$ and addictive state $s$, let $p_{s}^{a}$ denote the probability that environmental cues trigger the hot mode. Ordinarily, with continued use, the brain learns to make stronger associations between cues and the "high" produced by the substance, which suggests that $p_{s}^{a}$ rises with $s$. By assumption, the brain cannot enter the hot mode from the virgin state $\left(p_{0}^{a}=0\right)$. However, once the DM has been exposed to the substance, a myriad of (unconscious) cues, from a smell to a T.V. commercial, can potentially trigger the hot mode. We summarize our assumptions concerning the likelihood of entering the hot mode as follows:

Assumption 1: $p_{s}^{E}>p_{s}^{A} \geq p_{s}^{R}>0, p_{s+1}^{a} \geq p_{s}^{a}$, and $p_{0}^{a}=0$.

In this setting, individuals who enter the hot mode experience cravings; their attention is focused on getting the drug and on experiencing the "high". By assumption 1, 
rehabilitation can serve as either a method of precommiting to non-use despite an undiminished likelihood of experiencing cravings $\left(p_{s}^{R}=p_{s}^{A}\right)$, a strategy for avoiding cues that trigger cravings $\left(p_{s}^{R}<p_{s}^{A}\right)$, or both. As mentioned previously, rehabilitation does not preclude cravings in practice, and indeed addicts frequently enter rehabilitation to stop themselves from using substances when cravings arise.

At the end of each period, the DM spends his available resources $\left(y_{s}-r_{s}\right.$ if he has chosen $R$, and $y_{s}$ otherwise) on ordinary expenditures, $e$, and expenditures on the substance, $q x$ (where $q$ is the price of the substance, and $x$ is the quantity consumed). For simplicity we assume that the substance is only consumed at two levels, $x \in\{0,1\}$, and that the DM cannot borrow or save. When the DM elects $R$ at the outset of the period, he is constrained to choose $x=0$.

The brain assigns an instantaneous hedonic "payoff" $w_{s}(e, x, a)$ based on consumption ( $e$ and $x$ ), the activity chosen at the outset of the period (a), and the DM's addictive state $(s)$. We take $w_{s}$ to be strictly increasing in $e$, and we impose additional restrictions below in assumption 2. The dependence of the payoff function (and income) on the addictive state incorporates the effect of past usage on current wellbeing, including the effect of hedonic tolerance and of any health and socioeconomic costs of substance use. When pondering the desirability of any possible set of current and future outcomes, the DM always discounts future payoffs at a constant rate $\delta$ (irrespective of his cognitive state).

Note that the DM's instantaneous payoff is assumed to be independent of $\mu$. This implies that when a cue triggers cravings, the DM enters a hot cognitive mode, but his preferences do not change. This contrasts with the more conventional assumption that cravings are equivalent to cue-triggered changes in tastes (Laibson [2001]). In practice, the same cues that trigger the hot cognitive mode may also affect preferences. In this case, $\mu$ would enter as an argument of $w_{s}$, and utility would be state-dependent. Although this would complicate the problem somewhat, it would not inject any fundamental analytic difficulties. We nevertheless focus on the case in which $w_{s}$ is invariant with respect to cues because we can more clearly elucidate the implications of characterization failure by studying the phenomenon in isolation, rather than in combination with hedonic effects.

At this point, it is useful to provide some simplified notation. The individual's budget constraint requires $e+q x+r_{s}=y_{s}$. Define $u_{s}^{a} \equiv w_{s}\left(y_{s}, 0, a\right) ; b_{s}^{a} \equiv w_{s}\left(y_{s}-\right.$ $q, 1, a)-u_{s}^{a}$ for $a \in\{E, A\}$, and $c_{s} \equiv u_{s}^{R}-w_{s}\left(y_{s}-r_{s}, 0, R\right) \geq 0$ (with strict inequality when $r_{s}>0$ ). Intuitively, $u_{s}^{a}$ represents the baseline payoff associated with successful abstention in state $s$ and activity $a, b_{s}^{a}$ represents the marginal instantaneous benefit from use that the individual receives in state $s$ after taking activity $a$, and $c_{s}$ represents the cost of rehabilitation. Thus, $u_{s}^{a}+b_{s}^{a}$ is the payoff for usage, and $u_{s}^{R}-c_{s}$ is the payoff 
associated with rehabilitation. Let $p_{s}=\left(p_{s}^{E}, p_{s}^{A}, p_{s}^{R}\right), u_{s}=\left(u_{s}^{E}, u_{s}^{A}, u_{s}^{R}\right), b_{s}=\left(b_{s}^{E}, b_{s}^{A}\right)$, $\theta_{s}=\left(p_{s}, u_{s}, b_{s}, c_{s}\right)$, and $\theta=\left(\theta_{0}, \ldots, \theta_{S}\right)$ (likewise for $p, u, b$, and $c$ ). The vector $\theta$ specifies all the parameters of the consumption problem. These parameters are affected by the properties of the substance, the method of administration, the characteristics of the individual user, and the public policy environment. In keeping with our earlier discussion, we assume that:

Assumption 2: $u_{s}^{E}>u_{s}^{A} \geq u_{s}^{R}$ and $b_{s}^{E} \geq b_{s}^{A}$.

We also sometimes assume that the substance in question has the following additional properties:

Definition A substance is destructively addictive if, for all $a$ and $s=0, \ldots, S-1$, we have $b_{s+1}^{a} \geq 0, p_{s}^{a} \leq p_{s+1}^{a}, u_{s}^{a} \geq u_{s+1}^{a}, u_{s}^{a}+b_{s}^{a} \geq u_{s+1}^{a}+b_{s+1}^{a}$, and $c_{s} \leq c_{s+1}$.

A destructively addictive substance generates an immediate "high," except possibly in state 0 (see the discussion of acquired tastes below). The probability of entering the hot mode and the cost of rehabilitation increase with the addictive state. The payoffs associated with both abstention and use declines (possibly due to the mechanisms that produce tolerance). In contrast to other theories which assume that $b_{s}$ in increasing, here it may increase, decrease, or remain constant with $s$.

As mentioned at the outset of this section, we model characterization failure by assuming that the DM always chooses to consume the substance when $\mu=H$. One can imagine a number of plausible underlying cognitive mechanisms: environmental cues may induce the brain to focus attention on options involving use of the substance, or to ignore a variety of consequences other than the pleasure of the high. The particular mechanism is unimportant from our perspective. The key assumption here is simply that, when in the hot mode, the brain systematically mischaracterizes the DM's opportunity set in a way that induces him to consume the addictive substance.

By contrast, in the cold mode, the DM considers all possible courses of action and perfectly forecasts all future consequences, including the probability of entering the hot mode, which may lead to unwanted usage. Under these assumptions, the operations of the brain in the cold can be modeled as a simple dynamic stochastic programming problem. Maximization of discounted expected utility in each addictive state yields a value function $V_{s}(\theta)$ (measured as of the beginning of a period). For each state, there are five possible contingent plans available to the DM: engage in activity $E$ and then use the substance when in the cold mode $((a, x)=(E, 1))$, engage in $E$ and refrain from use when in the cold mode $((a, x)=(E, 0)$, henceforth "half-hearted abstention"), engage in $A$ and use when in in the cold mode $((a, x)=(A, 1))$, engage in $A$ and refrain from use when in the cold mode $((a, x)=(A, 0)$, henceforth "concerted abstention"), 
or enter rehabilitation $((a, x)=(R, 0))$. For $s \geq 1$, the expected payoffs associated with each of these contingent plans are as follows: ${ }^{33}$

$$
\begin{gathered}
\lambda_{s}^{a, 1}=u_{s}^{a}+b_{s}^{a}+\delta V_{\min \{S, s+1\}}(\theta) \text { for } a=E, A \\
\lambda_{s}^{a, 0}=u_{s}^{a}+p_{s}^{a} b_{s}^{a}+\left(1-p_{s}^{a}\right) \delta V_{\max \{\mathbf{1}, s-1}(\theta)+p_{s}^{a} \delta V_{\min \{S, s+1\}}(\theta) \text { for } a=E, A \\
\lambda_{s}^{R, 0}=u_{s}^{R}-c_{s}+\delta V_{\max \{\mathbf{1}, s-1\}}(\theta)
\end{gathered}
$$

Moreover, the value function must satisfy the following condition for each state $s$ :

$$
V_{s}(\theta)=\max _{(a, x) \in\{(E, \mathbf{1}),(E, 0),(A, \mathbf{1}),(A, 0),(R, 0)\}} \lambda_{s}^{a, x}
$$

Note that the parameter $p_{s}^{R}$ does not appear in the equations defining the value functions. Since, by assumption, cognitive modes are hedonically neutral, the parameter $p_{s}^{R}$ has no effect on the DM's choice, nor on his well-being.

We conclude this section with a few remarks about the model. First, it reduces to a standard problem when $p_{s}^{a}=0$ for all $s$. As discussed in the previous section, this may be a reasonable assumption for substances that do not impact the neural mechanisms governing incentive salience (that is "wanting" as opposed to "liking") with the same strength as drugs. Second, rehabilitation does not serve any purpose in this model other than pre-commitment. ${ }^{34}$ Third, we have assumed that the DM can commit to rehabilitation only one period at a time. Since the DM starts each period in the cold mode, this is without loss of generality.

\section{Positive Analysis}

We characterize the solution to the DM's optimization problem in the next two subsections. We begin by describing optimal choices within a period when continuation payoffs are governed by a given value function $V_{s}(\theta)$. We then explore the properties of the optimized value function and the associated decision functions. The remaining subsections examine implications for use.

\footnotetext{
${ }^{33}$ The associated valuation expressions for $s=\mathbf{0}$ are virtually identical, except that $V_{0}(\theta)$ replaces $V_{\max \{1, s-1\}}(\theta)$.

${ }^{34}$ In practice, rehabilitation programs may also teach self-management skills and desensitize addicts to cues. By practicing self-management skills, an addict may be able to alter the thoughts and images that the brain activates during the hot mode. Similarly, desensitization decreases the probability of entering the hot mode at any usage state. One can model these possibilities by assuming that $p_{s}$ (for a given state or states) declines subsequent to rehabilitation or therapy. Since the evidence suggests that these treatments are not completely effective (Goldstein [2001,p.188]), the forces described here would still come into play after treatment.
} 


\subsection{Optimal choice within a period}

Suppose that $V_{s}(\theta)$ describes continuation payoffs from the next period forward, and consider the DM's choice problem in any state $s$. It is easy to check that the DM never selects $(A, 1)$. This is intuitive: if he intends to consume the substance, there is no cost associated with exposure to cues that trigger the hot cognitive state.

For notational convenience, define

$$
\begin{aligned}
\Delta V_{s}(\theta) & =V_{\max \{\mathbf{1}, s-\mathbf{1}\}}(\theta)-V_{\min \{S, s+1\}}(\theta) \\
\mu_{s}(E, 1) & =\frac{b_{s}^{E}}{\delta}, \\
\mu_{s}(A, 0) & =\frac{\left(u_{s}^{E}-u_{s}^{A}\right)+\left(p_{s}^{E} b_{s}^{E}-p_{s}^{A} b_{s}^{A}\right)}{\delta\left(p_{s}^{E}-p_{s}^{A}\right)} \\
\mu_{s}(R, 0) & =\frac{b_{s}^{E}}{\delta}+\frac{u_{s}^{E}-u_{s}^{R}+c_{s}}{\delta p_{s}^{E}} \\
\mu_{s}^{A}(R, 0) & =\frac{b_{s}^{A}}{\delta}+\frac{u_{s}^{A}-u_{s}^{R}+c_{s}}{\delta p_{s}^{A}} .
\end{aligned}
$$

$\Delta V_{s}(\theta)$ measures the incremental future cost of usage in the current period. The constant $\mu_{s}(a, x)$ defines the value of $\Delta V_{s}(\theta)$ for which the DM is indifferent between $(a, x)$ and $(E, 0)$ (that is, $\lambda_{s}^{a, x}-\lambda_{s}^{E, 0}=0$ ). The constant $\mu_{s}^{A}(R, 0)$ defines the value of $\Delta V_{s}(\theta)$ for which the DM is indifferent between $(R, 0)$ and $(A, 0)$.

Simple algebraic manipulation of equations (1) through (3) reveals that the DM's set of optimal choices in state $s, \chi_{s}(\theta)$, satisfies:

$(E, 1) \in \chi_{s}(\theta)$ if and only if $\Delta V_{s}(\theta) \leq \mu_{s}(E, 1)$

$(E, 0) \in \chi_{s}(\theta)$ if and only if $\Delta V_{s}(\theta) \in\left(\mu_{s}(E, 1), \min \left\{\mu_{s}(A, 0), \mu_{s}(R, 0)\right\}\right)$;

$(A, 0) \in \chi_{s}(\theta)$ if and only if $\mu_{s}(A, 0) \leq \mu_{s}(R, 0)$ and $\Delta V_{s}(\theta) \in\left(\mu_{s}(A, 0), \mu_{s}^{A}(R, 0)\right)$;

$(R, 0) \in \chi_{s}(\theta)$ if and only if either $\mu_{s}(A, 0) \leq \mu_{s}(R, 0)$ and $\Delta V_{s}(\theta) \geq \mu_{s}^{A}(R, 0)$, or $\mu_{s}(A, 0) \geq \mu_{s}(R, 0)$ and $\Delta V_{s}(\theta) \geq \mu_{s}(R, 0) ;$

These conditions are summarized in figure 1. It is easy to check that assumptions 1 and 2 imply that $0<\mu_{s}(E, 1)<\min \left\{\mu_{s}(A, 0), \mu_{s}(R, 0)\right\}$, and $\mu_{s}(A, 0) \leq \mu_{s}(R, 0)$ iff $\max \left\{\mu_{s}(A, 0), \mu_{s}(R, 0)\right\} \leq \mu_{s}^{A}(R, 0)$. Thus, as shown in the figure, there are two possible cases, defined according to whether $\mu_{s}(R, 0) \lessgtr \mu_{s}(A, 0)$. In both cases the DM selects $(E, 1)$ for low values of $\Delta V_{s}(\theta)$, either concerted or half-hearted abstention for intermediate values, and $(R, 0)$ for sufficiently large values. This is intuitive: since $\Delta V_{s}(\theta)$ measures the future costs of current use, the DM is more likely to consume when $\Delta V_{s}(\theta)$ is lower. Note that whole-hearted abstention is possible only in case 2 .

Figure 2 illustrates the relation between the value function and the decision rule. Consider a substance for which the value function is decreasing in $s$ (as shown in 
theorem 4 below, this property holds for any destructively addictive substance) and $\mu_{s}(A, 0)<\mu_{s}(R, 0)$. The term $\Delta V_{s}(\theta)$ measures the "steepness" of the value function. The previous analysis implies that the DM uses the substance in states for which the value function is flat, enters rehabilitation in states for which it is steep, and attempts to abstain (either half-heartedly or concertedly) for intermediate cases.

In general, $b_{s}^{E}<0$ does not rule out intentional consumption of the substance in state $s$ (that is, $(E, 1)$ ). This can occur if the value function is increasing in the state s. When $b_{0}^{E}<0$ and $(E, 1) \in \chi_{0}(\theta)$, we say that the substance gives rise to an acquired taste. Certain methods of consuming alcohol and nicotine (e.g. beer and cigars) are sometimes identified as examples of this phenomenon.

\subsection{Dynamic optimization}

In the previous section, we characterized optimal choices for an arbitrary value function. We now turn our attention to the properties of the optimized value function, and we explore implications for optimal dynamic choice.

Since the model is formulated at a reasonably high level of generality, we are unable to provide closed-form analytic solutions. Instead, we characterize the directional effect of each parameter on the value function and on optimal usage. We begin with a simple result concerning the value function. ${ }^{35}$

Theorem 1: For all $s, V_{s}(\theta)$ is continuous in $\theta$, weakly increasing in $u_{k}^{E}, u_{k}^{A}, u_{k}^{R}$, $b_{k}^{E}$, and $b_{k}^{A}$, and weakly decreasing in $p_{k}^{E}, p_{k}^{A}$, and $c_{k}$.

This result is intuitive. When $b_{k}^{a}$ or $u_{k}^{a}$ increase, or when $c_{k}$ decreases, the same decision rule must yield weakly higher valuations for every state $s$; hence, $V_{s}(\theta)$ cannot decline. A slightly different argument is needed to establish the monotonicity with respect to $p_{k}^{a}$.

Henceforth, we will say that usage in state $s$ is (weakly) increasing in a parameter if an increase in the parameter leads the DM to choose, for that state, a course of action associated with a higher probability of usage. ${ }^{36}$ Recall that $(E, 1)$ is associated with the highest probability of usage, followed (in order) by $(E, 0),(A, 0)$, and $(R, 0)$ (the DM never chooses $(A, 1))$.

As shown in the preceding section, optimal choices depend not upon the absolute size of $V_{s}(\theta)$ in any state $s$, but rather on the differences in valuation across states (that

\footnotetext{
${ }^{35} \mathrm{As}$ mentioned previously, $p_{k}^{R}$ is an irrelevant parameter.

${ }^{36}$ Since the optimal action in any state need not be unique, a technical clarification is required. We say that usage in state $s$ is higher with parameter vector $\bar{\theta}$ than with $\underline{\theta}$ if any element of the (possibly empty) set $\chi_{j}(\underline{\theta}) \backslash \chi_{j}(\bar{\theta})$ involves a lower probability of usage than any element of $\chi_{j}(\bar{\theta})$, and any element of the (possibly empty) set $\chi_{j}(\bar{\theta}) \backslash \chi_{j}(\underline{\theta})$ involves a higher probability of usage than any element of $\chi_{j}(\underline{\theta})$.
} 
is, $\Delta V_{s}(\theta)$ ). Consequently, the critical question with respect to behavior is not whether a change in some particular parameter raises or lowers $V_{j}(\theta)$, but rather whether the absolute change in valuation is larger in some states than in others. For this reason, Theorem 1 might at first appear to be of limited use with respect to characterizing behavioral responses to changes in parameters. On the contrary, the result turns out to be extremely useful. In the appendix, we show that a change in an element of $\theta_{k}$ (the parameters affecting the instantaneous payoff in the addictive state $k$ ) has a larger effect on the value function for states that are closer to $k$ (see lemma 1). Consequently, if the value function is increasing in a particular state $k$ parameter, an increase in this parameter increases $\Delta V_{j}(\theta)$ for $j<k$, and reduces $\Delta V_{j}(\theta)$ for $j>k$ (see lemma 2 in the appendix). It then follows from the analysis in the previous section that usage increases in states $j<k$, and decreases in states $j>k$. Supplementing this line of proof with some additional arguments, we obtain a reasonably complete characterization of comparative dynamics:

Theorem 2: Usage in state $j$ is:

(i) weakly increasing in $b_{k}^{a}$ and $u_{k}^{a}$, and weakly decreasing in $p_{k}^{a}$ and $c_{k}$, for $k>j$,

(ii) weakly decreasing in $b_{k}^{a}$ and $u_{k}^{a}$, and weakly increasing in $p_{k}^{a}$ and $c_{k}$, for $k<j$,

(iii) weakly decreasing in $p_{j}^{E}$ and $u_{j}^{R}$ and weakly increasing in $b_{j}^{E}$ and $c_{j}$.

This theorem establishes that use in state $s$ is monotonic with respect to most parameters and indicates the direction of the effect. The only exceptions concern the effects of $b_{j}^{A}, u_{j}^{A}$, and $u_{j}^{E}$ on usage in state $j$, which can be positive or negative, depending on the parameter values. ${ }^{37}$ Interestingly, while changes in $p_{k}^{E}$ and $c_{k}$ affect usage in states $j \neq k$ in the same direction, they have opposite effects in state $k$.

Theorem 2 underscores the fact that policy changes can have complicated behavioral effects. For example, a policy that reduces usage in the late stages of addiction by decreasing the cost of rehabilitation may also increase use and discourage rehabilitation at earlier stages. This effect may be particularly strong when subsidized rehabilitation is only offered to the most serious addicts. Indeed, an increase in the cost of rehabilitation for highly addicted states may unambiguously reduce both total use, and use at higher states, by inducing a shift to rehabilitation at an earlier state. This is an argument for early intervention. Similarly, a reduction in $p_{k}^{a}$ reduces unintended usage, but it increases intentional usage among new users.

\footnotetext{
${ }^{37}$ An increase in $b_{j}^{A}$ or $u_{j}^{A}$ can shift the optimal state $j$ choice from either $(E, \mathbf{0})$ or $(R, 0)$ to $(A, \mathbf{0})$. An increase in $u_{j}^{E}$ can induce a shift from $(E, 1)$ to $(E, 0)$ in state $j$ if, for example, $(E, 1)$ is optimal in states $j-1$ and $j+1$. It can also induce a shift from $(E, 0)$ to $(E, 1)$ in state $j$ if, for example, $(R, 0)$ is optimal in states $j-1$ and $j+1$.
} 
Theorem 2 considers the effects of changing parameters for one addictive state at a time. To compare behavior across different users, substances, methods of administration, or policy regimes, it is often necessary to consider alternative parameter vectors that differ in all addictive states. Fortunately, theorem 2 facilitates such comparisons. The following corollary provides an illustration.

Corollary: Consider some $\theta$ derived from $w_{s}(e, x, a)$, and $\theta^{\prime}$ derived from $w_{s}^{\prime}(e, x, a)=$ $w_{s}(e, x, a)+d_{s}$.

(i) If, for some $k$, we have $d_{k} \leq d_{s}$ for $s<k$ and $d_{k} \geq d_{s}$ for $s>k$, then usage in state $k$ is weakly higher with $\theta$ than with $\theta^{\prime}$.

(ii) If $d_{s}$ is weakly decreasing in $s$, then usage is weakly higher with $\theta$ than with $\theta^{\prime}$ for all states $s$.

The corollary describes the manner in which usage varies with the pattern of baseline well-being over addictive states. It allows for the possibility that $\theta$ differs from $\theta^{\prime}$ in all states. Nevertheless, it derives the following unambiguous prediction (part (ii)): when there is greater deterioration of baseline well-being as the addictive state increases, usage is lower in all states. To prove part (i), consider $\theta^{\prime \prime}$ derived from $w_{s}^{\prime}(e, x, a)=w_{s}(e, x, a)+d_{k}$. Clearly, usage is identical for $\theta$ and $\theta^{\prime \prime}$ (utility differs only by a constant). But theorem 2 implies that usage in state $k$ is higher for $\theta^{\prime \prime}$ than for $\theta^{\prime}$. To prove part (ii), note that the condition in part (i) is satisfied for all $s$ whenever $d_{s}$ is weakly decreasing in $s$.

The previous results provide conditions under which changes in the parameters produce monotonic changes in behavior. By contrast, the next result identifies circumstances in which parameter changes have no effect on the optimal decision (or the value function).

Theorem 3: $\quad \chi_{j}(\theta)$ and $V_{j}(\theta)$ are invariant with respect to:

(i) any changes in $p_{j}^{E}, p_{j}^{A}, u_{j}^{A}, u_{j}^{R}, b_{j}^{A}$, and $c_{j}$ when $(E, 1) \in \chi_{j}(\theta)$

(ii) any increase in $p_{k}^{a}$ and $c_{k}$ or any decreases in $b_{k}^{a}$ and $u_{k}^{a}$ when $k>j$ and $(R, 0) \in \chi_{n}(\theta)$ for some $n \in\{j, \ldots, k-1\}$

(iii) any increase in $p_{k}^{a}$ and $c_{k}$ or any decreases in $b_{k}^{a}$ and $u_{k}^{a}$ when $k<j$ and $(E, 1) \in \chi_{n}(\theta)$ for some $n \in\{k+1, \ldots, j\}$

Part (i) states that, if $(E, 1)$ is optimal in state $j$, then no (global or local) change in any of the listed parameters (subject to the restrictions of assumptions 1 and 2) can affect behavior in state $j$. Strikingly, one cannot induce a state $j$ user to enter rehabilitation by reducing the state $j$ rehabilitation cost. ${ }^{38}$ Similarly, one cannot induce

${ }^{38}$ However, one can induce a state $j$ user to accept rehabilitation by paying him to enter rehabilitation, a possibility we have ruled out by assuming that $r_{j} \geq \mathbf{0}$. 
a state $j$ user to abstain by reducing the state $j$ probability of entering the hot mode. Part (ii) of theorem 3 states that a (global or local) increase in $p_{k}^{a}$ or $c_{k}$, or a (global or local) decrease in $u_{k}^{a}$ or $b_{k}^{a}$, have no effect on the usage or welfare of an earlier state $j$ when there is an intermediate state $n \in\{j, \ldots, k-1\}$ for which rehabilitation is optimal. Part (iii) is similar to part (ii).

We close this section with an intuitive result concerning destructively addictive substances. In particular, for any such substance, the DM's optimized well-being declines monotonically with the addictive state. It follows immediately that the DM never intentionally acquires a taste for a destructively addictive substance.

Theorem 4: For any destructively addictive substance, $V_{s}(\theta) \geq V_{s+1}(\theta)$ for all $s$. Moreover, $b_{0}^{E}<0$ implies $(E, 1) \notin \chi_{0}(\theta)$.

\subsection{Patterns of use}

Since the model generates a mapping from parameters (which depend upon the characteristics of the user, substance, method of administration, and policy regime) to consumption patterns, it is possible in principle to confront the model with data and to test its implications. Although empirical work is beyond the scope of the current paper, in this section we show through a series of examples that the model generates a broad range of consumption patterns that are observed in practice, as well as a plausible qualitative mapping between parameters and behavior. This discussion is based on robust numerical examples which, given the length of the paper, are omitted.

\subsubsection{Use and non-use}

Certain substances are associated with consistent use. Caffeine is a familiar example. The key characteristics of caffeine are as follows: it produces a "high" in every state $\left(b_{s}>0\right)$; it slowly generates tolerance and withdrawal symptoms with sudden abstention; and, if consumed in large amounts, it gradually creates long-term adverse health consequences such as anxiety and sleep disturbances (see Goldstein [2001,ch.13]). To understand the implications of our model for a substance such as caffeine, consider first a hypothetical substance that confers a constant positive benefits $\left(b_{s}=b_{0}>0\right)$ and for which utility is independent of the addictive state $\left(w_{s}(e, x, a)=w(e, x, a)\right.$ for all

$s)$. In this case, $(E, 1)$ is the optimal choice in all states. This is intuitive: usage is optimal when it is pleasurable and there are no future consequences. By an analogous argument, consistent use remains optimal as long as the parameters $\theta_{s}$ do not change much from one state to the next, as in the case of caffeine. In this instance, $\Delta V_{s}(\theta)$ is small and one still has $\Delta V_{s}(\theta)<\mu_{s}(E, 1)=\frac{b_{s}^{E}}{\delta}$. For such a substance, well-being may nevertheless decline significantly from state 0 to state $S$. 
In contrast, the model produces non-use (meaning that the DM does not select $(E, 1)$ in any state) whenever $\Delta V_{s}(\theta)>\mu_{s}(E, 1)=\frac{b_{s}^{E}}{\delta}$ for all $s$. This occurs for any substance (or user) that produces a sufficiently mild high ( $b_{s}^{E}$ small) or sufficiently harmful consequences (for example, $w_{s}(e, x, a)=w(e, a)+d_{s}$, where $d_{s}$ is steeply decreasing in $s$ ). The DM never begins using such a substance and, if placed in an addictive state $s>0$, he does not use intentionally.

Characterization failure plays no role in explaining consistent use. It is easy to check that, if $(E, 1)$ is optimal in all addictive states, then the DM elects consistent use irrespective of the probability parameters $p_{s}^{a}$. In contrast, characterization failure can play an important role in explaining non-use. An individual may choose not to use a substance in some state because he fears the likelihood of cue-induced usage in more advanced addictive states. In particular, many people may refrain from experimenting with "hard drugs" because they fear that frequent use could produce a downward spiral of addiction.

\subsubsection{Intermittent use and forms of abstention}

For substances such as alcohol and cocaine, the deleterious consequences of consumption (e.g. health effects and interference with normal personal and social activities) are greater for heavy users than for light users. Within the context of our model, this characteristic produces many of the usage patterns associated with these substances.

Assume in particular that $u_{s}^{a}$ and $u_{s}^{a}+b_{s}^{a}$ decline with $s$, and that the rate of decline accelerates with $s$. The associated value function tends to inherit these properties. Assuming that the incremental benefits of use, $b_{s}^{a}$, are relatively constant, the resulting increase in $\Delta V_{s}(\theta)$ across addictive states tends to shift the DM toward abstention. If the increase is sufficiently gradual, a region of use (states $s$ such that $\Delta V_{s}(\theta)<$ $\left.\mu_{s}(E, 1)\right)$ is followed by a region in which the DM selects activity $E$ with the intention of abstaining (states $s$ such that $\Delta V_{s}(\theta) \in\left(\mu_{s}(E, 1), \min \left\{\mu_{s}(A, 0), \mu_{s}(R, 0)\right\}\right)$ ). This gives rise to intermittent use. The DM is initially attracted to the substance because it delivers an enjoyable high, and because light use is relatively innocuous. However, as repeated use begins to take its toll, the individual decides that moderation is desirable.

To illustrate, suppose that $S=4$ and the optimal choices are $(E, 1)$ for states 0 and 1 , and $(E, 0)$ for states 2 through 4 (see the left half of figure 2, states 0 through 4 ). In that case, the DM chooses to start using the substance, and continues using it for a second period. Subsequently, provided that he remains in the cold cognitive mode, he uses the substance in alternate periods, moving back and forth between states 1 and 2. Of course, with some probability, the DM encounters environmental cues that trigger the hot cognitive mode, which results in use of the substance. This moves him to a higher addictive state, where the likelihood of triggering the hot mode is 
even greater. In other words, the intermittent user is susceptible to cue-conditioned cravings, which can set off unintended binges. Indeed, if he encounters a sequence of environmental cues, he may find himself in state 4 after using the substance against his better judgement for several consecutive periods. Over time, the DM settles into a stochastic steady state, distributing his time between states 1 to $4 .^{39}$

In the previous example, the DM always engages in half-hearted abstention: e.g. an alcoholic intends to abstain, but nevertheless attends a party at which alcohol is readily available, knowing that the temptation to indulge may be impossible to resist. If $\Delta V_{s}(\theta)$ continues to increase gradually over addictive states, and if $\mu_{s}(A, 0)<\mu_{s}(R, 0)$ for the relevant states, a region in which the DM selects $E$ with the intention of abstaining may be followed by a region in which he selects activity $A$ with the intention of abstaining (states $s$ such that $\Delta V_{s}(\theta) \in\left(\mu_{s}(A, 0), \mu_{s}^{A}(R, 0)\right)$ ). In comparison to $E$, action $A$ is less intrinsically enjoyable, but reduces the likelihood of encountering environmental cues that trigger the hot cognitive mode. Thus, one can think of the course of action $(A, 0)$ as concerted abstention through cue avoidance (e.g. choosing to read a book at home rather than attend the party).

To illustrate the resulting consumption patterns, modify the preceding example by adding two additional states, $s=5,6$, in which, as depicted in figure 2, the DM selects $(A, 0)$. Once again, the DM chooses to start using the substance, and continues using it for a second period. He then engages in half-hearted abstention, which may or may not be successful. For a time, he bounces between states 2 and 4, in some instances consuming intentionally, and in others attempting half-hearted abstention with varying success. With the passage of sufficient time, he inevitably finds himself in state 5, wherein the consequences of continued use are more severe. At this point, he becomes more determined to take a break from the substance, and chooses to avoid the settings in which he encounters environmental cues that trigger usage.

\subsubsection{Rehabilitation and recidivism}

In practice, alcohol and substance abusers tend to seek treatment (rehabilitation) when they view the consequences of continued use as sufficiently dire, and when they despair of controlling their behavior without assistance. Within the context of our model, one can depict the first characteristic by assuming, as before, that $u_{s}^{a}$ and $u_{s}^{a}+b_{s}^{a}$ decline rapidly with $s$ among heavy users. One can depict the second characteristic by assuming that the probability of triggering the hot mode, $p_{s}^{a}$, is substantial for heavy users. Under the first assumption, $\Delta V_{s}(\theta)$ is large; under the second assumption,

\footnotetext{
${ }^{39}$ The tendency for unintended consumption to produce binging is even more pronounced if, once in the hot cognitive state, the DM temporarily becomes more susceptible to environmental cues. To model this effect, one could allow $p_{s}^{a}$ to depend upon $\mu_{t-1}$ (that is, assume that the probability of the hot cognitive mode is given by $p^{a}\left(s_{t}, \mu_{t-1}\right)$, with $\left.p^{a}(s, H)>p^{a}(s, C)\right)$.
} 
$\mu_{s}(R, 0)$ and $\mu_{s}^{A}(R, 0)$ are small. With this combination of conditions, our model produces realistic usage patterns involving rehabilitation and recidivism.

When $\Delta V_{s}(\theta)$ rises gradually and/or the pertinent threshold (either $\mu_{s}(R, 0)$ and $\left.\mu_{s}^{A}(R, 0)\right)$ declines gradually with $s$, the DM chooses to enter rehabilitation only after unsuccessfully attempting to abstain. This is illustrated once again in figure 2 (states 0 to 8 only). For concreteness, assume that $S=8$, that the optimal choices are as before for states 0 to 6 , and that the DM selects $(R, 0)$ for states 7 and 8 . Once again, the DM chooses to start using the substance, and continues using it for a second period. He then attempts half-hearted abstention. If this is unsuccessful, the adverse consequences of continued use increase, so he makes a concerted attempt to abstain. If this is also unsuccessful, his well-being becomes even more vulnerable to continued use, so he enters a rehabilitation clinic.

When the DM emerges from rehabilitation, he resumes his attempt to abstain (concertedly) from use. If he is successful, his addictive state declines, and his efforts to abstain become half-hearted. With continued success, he may resume intermittent use, bouncing between addictive states 1 and 2. However, as long as $p_{s}^{A}>0$ for all $s$, the DM returns to rehabilitation in finite time with probability one. Thus, the model gives rise (inevitably) to long-term recidivism even when rehabilitation is followed by short-term abstention.

When the rise in $\Delta V_{s}(\theta)$ or the change in the pertinent threshold is sufficiently sharp, the DM chooses to enter rehabilitation without passing through addictive states in which he attempts to abstain. That is, one can have situations in which, for example (with $S=5$ ), optimal choices are $(E, 1)$ for states 0 through 3 and $(R, 0)$ for states 4 and 5. One natural way to construct such an example is to assume that $b_{s}^{a}$ declines sharply between two consecutive addictive states. In such cases, the model produces cycling between use and rehabilitation. The DM enters rehabilitation in each instance without any desire to stay clean; he knows that he will resume using the substance upon release from rehabilitation, and fully expects to enter rehabilitation once again. We refer to this pattern as intentional recidivism. It is in fact observed among serious heroin users when repeated use dilutes the "high" (see Massing [2000]). This is evidence of fairly sophisticated, forward thinking among junkies whose objective is to renew the high by temporarily getting clean. Characterization failure plays a perverse role in producing in this pattern: the DM checks into a rehabilitation clinic because there is a risk that he might not be able to abstain on his own, decreasing even further his ability to experience the high. 


\subsubsection{Resignation}

Even for extremely harmful substances such as crack and heroin, the damage associated with incremental usage may level off once social networks have been destroyed, jobs have been lost, and poor health has become the norm. Within the context of our model, one can depict this characteristic by assuming that the decline in $u_{s}^{a}$ and $u_{s}^{a}+b_{s}^{a}$ decelerates for the heaviest users. In that case, the value function flattens out for large $s$, as shown in the right half of figure 2. If the adverse consequences of usage are also initially slow to develop, then the value function $V_{s}(\theta)$ tends to inherit the "inverted- $S$ " shape shown in the figure. At intermediate addictive states, the DM may engage in concerted abstention, or even enter rehabilitation. If, due to adverse circumstances, his addictive state continues to rise, his efforts to abstain may become increasingly half-hearted. When he reaches states for which $V_{s}(\theta)$ is sufficiently flat, the addict returns to intentional consumption. This occurs because the incremental harm from usage and the probability of successful abstention are both sufficiently low. We refer to this pattern as resignation; the addict gives up, accepts failure, and "lets himself go." It is commonly observed among long-term crack and heroin users who settle into subsistence lifestyles at the fringes of society.

\subsubsection{Quitting}

As noted in section 2.1, users of addictive substances often attempt to quit permanently, but achieve limited success. Our model produces many of the patterns associated with quitting.

In some of the preceding examples, the courses of action $(E, 0)$ and $(A, 0)$ represent decisions to take a break from the substance, rather than to quit. In contrast, an individual in addictive state $s$ who wishes to quit using a substance would, in the cold mode, select a course of action other than $(E, 1)$ in every state $s \geq 1$. When continued use is sufficiently harmful (e.g. $w_{s}(e, x, a)=w(e, a)+d_{s}$, where $d_{s}$ decreases rapidly in $s$ ) and insufficiently pleasurable ( $b_{s}^{a}$ small), this pattern is optimal. ${ }^{40}$ To illustrate some phenomena associated with quitting, imagine again that $S=5$, that the optimal choices are $(E, 0)$ for states 1 through 2 and $(A, 0)$ for states 3 through 5 . This DM wishes to quit, in the sense that he always chooses not to consume when in the cold mode. If his state of addiction is advanced $(s \geq 3)$, he avoids the cues that trigger cravings. Once he successfully reduces his addictive state $(s<3)$, he allows himself to

\footnotetext{
${ }^{40}$ This does not rule out the possibility that $(E, 1)$ is optimal in state 0 , which explains why the individual first began to use the substance. Alternatively, one can assume that the individual's preferences changed since he starting using the substance, that he learned the true values of various parameters only after experimenting with the substance, or that his initial decision to use (e.g. as a youth) was irrational.
} 
engage in pleasurable activities that are more likely to trigger cravings, but continues to abstain whenever possible.

In our model, the success of any attempt to quit (as measured by the frequency of non-use) depends upon two factors. The first factor is the individual's underlying susceptibility to characterization failure, measured by $p_{s}^{a}$. As long as $p_{s}^{a}>0$ for $s \geq 1$, the individual never succeeds in quitting completely: there is always some probability of renewed use. Notably, this is one of the central tenets of Alcoholics Anonymous:

"So far as can be determined, no one who has become an alcoholic has ever ceased to be an alcoholic. The mere fact of abstaining from alcohol for months or even years has never qualified an alcoholic to drink 'normally' or socially. Once the individual has crossed the borderline from heavy drinking to irresponsible alcoholic drinking, there seems to be no retreat... [I]f you are an alcoholic, you will never be able to control your drinking for any length of time." 41

The second factor is the extent to which the individual is willing to engage in concerted abstention through cue avoidance. For the same probability parameters, the DM will plainly achieve greater success if he selects $(A, 0)$ in all addictive states, rather than in a subset of states, as in the preceding example. Avoidance of familiar environmental triggers is an important feature of many treatment programs.

Any individual who engages in either concerted or half-hearted abstention (whether to quit or take a break from use) will manifest a demand for attention management therapies. The purpose of such therapies is to divert attention from environmental cues that trigger the hot cognitive mode, to refocus attention on consequences that return the individual to the cold cognitive mode before he takes action, or to desensitize the individual to established cues. Without formally modeling the attention process, one can capture the effects of these therapies in a stylized way as a reduction in the state-and-action-specific probability of entering the hot cognitive mode (that is, $p_{s}^{a}$ ). According to theorem $1, V_{s}(\theta)$ is weakly decreasing in $p_{k}^{a}$. Moreover, it is easy to show that $V_{s}(\theta)$ is strictly decreasing in $p_{s}^{a}$ whenever $(a, 0)=\chi_{s}(\theta)$. In that case, the DM is willing pay a positive price (measured either as a reduction in $y_{s}$, or as a utility penalty reflecting time and effort) in return for therapy that reduces $p_{s}^{a}$.

\subsection{Other implications for addictive behavior}

Some of the stylized facts concerning addiction discussed in section 2.1 have formal counterparts within our analysis, while others do not. In particular, the consumption patterns described above demonstrate that the model can account for the first, second,

\footnotetext{
${ }^{41}$ See http://www.alcoholics-anonymous.org/english/E_Pamphlets/P-2_d1.htm
} 
third, fifth, and sixth stylized facts, as well as for the value of attention management therapies. In this section we argue that the model is also consistent with the remaining facts.

We have noted that addicts experience feelings of powerlessness. In our model, the individual sometimes consumes the substance despite intending to abstain. When the likelihood of experiencing cravings is sufficiently high, the intention to abstain rarely translates into action. It stands to reason that the anticipation of consistent failure would translate into a feeling of powerlessness.

Likewise, we have observed that addicts report feelings of regret and the sense that they are making mistakes even during the act of consumption, as well as the anticipation that they will continue to make mistakes in the future. Our theory is predicated on the notion that individuals anticipate future mistakes, and recognize that current actions can trigger those mistakes. With respect to consumption-in-progress, a user will experience concurrent regret if characterization failure is sufficiently short-lived (that is, if he returns to the cold mode immediately after administering the substance, while he is beginning to, or in the process of, enjoying the high). Stepping outside our simple model, one can also imagine a cognitive process through which the DM learns, and is at least peripherally aware at all times, that he tends to make poor decisions in the hot mode.

We have also observed that the effects of addictive substances vary considerably across users. In particular, only a fraction of those who experiment with drugs early in life become addicts. Our theory offers one possible explanation: those who are more susceptible to develop characterization failure, perhaps due to genetic differences, are more vulnerable to addiction. ${ }^{42}$ This explanation is consistent with the observation that brain circuitry differs systematically between addicts and non-addicts. ${ }^{43}$ If correct, it suggests a useful method for identifying young individuals at risk of addiction (e.g. through cognitive tests along the lines of the experiments conducted by Mischel and others, which we discussed in section 3.1). ${ }^{44}$ An important open question is whether the succeptibility to characterization failure is a general trait, which would explain addictive personalities, or something that is specific to the individual and substance, which would give rise to idiosyncratic vulnerabilities.

\footnotetext{
${ }^{42}$ See Goldstein [2001,ch. 7] for a discussion of the evidence of the role of genes in addiction.

${ }^{43}$ Using brain imaging technologies, Volkow (1997) and Volkow et. al. (1997) have shown that the brains of long-term methamphetamine users have fewer dopamine receptors than those of nonusers. This research does not, however, establish whether the difference is due to drug exposure or, as required by our hypothesis, to pre-existing neurological differences (or sensitivities).

${ }^{44}$ Longitudinal studies of preschoolers have shown that the length of time for which 4-year olds can delay gratification is correlated significantly with their performance, as adolescents, on standardized tests and parental ratings of competencies such as the ability to plan, exert self-control, and focus (Metcalfe and Mischel [1999]).
} 
Finally, we have noted that the behavior of some addicts is sensitive to reminders about the consequences of use (concerning, for example, the damage caused to his family, or the likelihood of dying from an overdose). Since a reminder, by definition, conveys no new information, it is difficult to explain this sensitivity within the context of the standard model. In contrast, reminders can influence the behavior of an individual whose attention and memory are affected by cues, by inducing the brain to activate thoughts about information that it already possesses. While our model does not formally depict the mechanisms by which reminders affect behavior, its foundations are consistent with the existence of these effects. One can introduce these considerations into our model in a stylized way by assuming that a reminder reduces the probability of entering the hot cognitive mode.

\subsection{Some extensions}

One unrealistic aspect of our model is that the DM always makes the same choice in each addictive state $s$. Once $s$ exceeds an addictive state in which the DM selects $(E, 1)$, it never falls below that state again. Similarly, the DM can never pass beyond a state in which $(R, 0)$ is chosen. Consequently, the DM eventually becomes trapped between the last state in which $(E, 1)$ is chosen and the first state in which $(R, 0)$ is chosen. One can overturn this property by introducing some additional uncertainty. To illustrate, imagine that $w_{s}(e, x, a)=w_{s}(e, x)+\eta_{a}$. Suppose that, at the outset of each period, $\eta=\left(\eta_{E}, \eta_{A}, \eta_{R}\right)$ is determined at random by factors affecting the DM's mood (stress, anxiety, etc.), and that the DM learns these values prior to making any decisions. Conditional on any realization of $\eta$, our static analysis is unchanged. Consequently, for any distribution of $\eta$, it is a simple matter to describe the choice in any state $s$ probabilistically. The dynamic analysis proceeds essentially as before (except that the value function is computed as an expectation), and most of our results continue to hold. With a sufficiently extreme realization of $\eta$, the DM can pass beyond or below any addictive state.

Another unrealistic assumption is that the DM knows all of the parameters of the problem prior to using the substance for the first time. In a more realistic model, the DM would learn a great deal through use (e.g. about the nature and intensity of the "high"). This provides a motive for experimentation. ${ }^{45}$ To illustrate, imagine that the individual is uncertain about the parameter vectors $b, u$, and $c$, and, for simplicity, that this uncertainty is entirely resolved when the individual uses the substance for the first time. Since the DM's expected discounted payoff is linear in $b, u$, and $c$

\footnotetext{
${ }^{45}$ Here we discuss experimentation with use. The DM may also have a motive to experiment with abstention in order to assess whether he is becoming (or likely to become) addicted. Hung [2000] and Orphanides and Zervos [1995] study experimentation in the context of the rational addiction model.
} 
for each complete contingent plan, the optimized value function $V_{s}(\theta)$ must be convex in these parameters (it corresponds to the upper envelope of linear functions). It follows that initial usage (weakly) increases when a mean-preserving spread is applied to the distributions of $b, u$, and $c$. Thus, although it is natural to conjecture that the individual would be less willing to experiment with a substance in the presence of greater uncertainty, this turns out to be incorrect.

This result has several practical implications. First, young people may be more likely to experiment with addictive substances than older people, even if age is unrelated to average tastes, and even if young people are no more likely to have biased expectations concerning long-term costs, merely because the young are less certain about their own tastes and proclivities. Second, experimentation with new substances ("fad drugs") is likely to be large simply because there is greater uncertainty about their effects. Third, information policies can discourage experimentation and use merely by reducing uncertainty about the effects of drugs, even if potential users already have unbiased expectations. Fourth, as discussed in section 5.3.5, individuals may rationally choose to begin using an addictive substance, and subsequently decide to quit without a change in preferences.

Our model can also be extended, or in some cases simply reinterpreted, to describe precommitment strategies other than rehabilitation. A particularly interesting strategy entails the use of the agonist, antagonist, and metabolic medications described in section 2.2. Under appropriate assumptions, one can interpret our model as encompassing this strategy. In particular, imagine that, at the beginning of each period, the individual chooses between three actions: take a dose of disulfiram $(A)$, do not take a dose of disulfiram $(E)$, or enter rehabilitation $(R)$. In this context, we assume that $b_{s}^{A}<0$ (consumption of alcohol becomes unpleasant) and $p_{s}^{A}<p_{s}^{E}$ (anticipation of unpleasant consequences makes it less likely that environmental cues will trigger cravings). ${ }^{46}$ Plainly, the DM will select alternative $A$ only if continued use is sufficiently damaging $\left(\Delta V_{s}(\theta)\right.$ large) and the reduction in the probability of unintended use is large enough to overcome the unpleasant effects of occasionally drinking after taking disulfiram.

In practice, compliance with the disulfiram treatment regimen is a significant problem (Goldstein [2001]). Conceivably, the failure to take disulfiram could be attributable to characterization failure. One could model this possibility by relaxing the assumption that the DM necessarily enters each period in the cold cognitive mode. In such a model, reducing the frequency with which the medication must be taken (e.g. to every other period) would increase its effectiveness at reducing usage of the addic-

\footnotetext{
${ }^{46}$ According to Goldstein [2001, p. 151], "an agent who, in the sober state, is motivated to take [disulfiram] regularly will be unlikely to succumb to the craving for a drink, knowing (perhaps from one bad experience) what is bound to happen."
} 
tive substance. In practice, therapeutic drugs with long-lasting effects produce lower frequencies of relapse than do drugs with more transient effects (O'Brien [1997]).

\section{Normative Analysis}

Since our formulation of preferences is completely standard, the appropriate measure of welfare is simply discounted experiential utility: $\sum_{t=0}^{\infty} \delta^{t} w_{s_{t}}\left(e_{t}, x_{t}, a_{t}\right)$. A policy can improve welfare if and only if it reduces the probability of entering the hot mode in an instance where this would lead to a suboptimal choice, or if it forces the agent to make a preferred choice while in the hot cognitive mode.

Notably, in the case of constant use (for which the DM's decisions in the hot mode and cold mode coincide), the laissez faire outcome is first-best, and no welfareimproving policy exists, irrespective of whether characterization failure occurs with high frequency. Thus, the development of a substance such as Aldox Huxley's fictional "soma" from the classic novel Brave New World, which causes the user to feel blissfully happy regardless of circumstances, would be welfare-improving according to the normative criterion adopted herein. We acknowledge that this implication is controversial.

In the remainder of this section, we study the welfare effects of various public policies concerning addictive substances. To focus our analysis on the implications of characterization failure, we restrict attention to "demand side" welfare effects, ignoring "supply side" consequences associated with the development of black markets, the spread of corruption, and enforcement costs. ${ }^{47}$ Our object is not to provide a complete normative analysis of drug policy. Instead, we investigate the extent to which various government policies benefit some users by helping them to overcome the adverse consequences of characterization failure, and harm others by distorting deliberate (rational) consumption. For simplicity, we assume throughout that the addictive substance is competitively produced using a constant-returns-to-scale technology, so that supply is infinitely elastic at a price equal to some marginal cost $q$. We also ignore potential consumption externalities. ${ }^{48}$ Since externalities provide a well-understood rationale for government intervention, this permits us to isolate the welfare and policy implications of characterization failure.

\footnotetext{
${ }^{47}$ Supply side effects are discussed elsewhere; see e.g. See McCoun and Reuter [2001] and Miron and Zwiebel [1995].

${ }^{48}$ There are three distinct classes of externalities to consider. First, the consumption of addictive substances may inflict costs on others directly (such as accidents due to drunk driving or the spread of infectious diseases). Second, for some substances, consumption is a social activity: a user enjoys larger benefits when others join in. Third, consumption by one individual may generate cues that trigger characterization failure for others. Note that the first and third considerations are external diseconomies, while the second is an external economy.
} 


\subsection{Taxation and subsidization}

A number of addictive substances (e.g. nicotine and alcohol) are subject to heavy taxation, and various forms of treatment are subsidized. It is therefore natural to examine the welfare effects taxes and subsidies.

When considering the welfare effects of such policies, one must specify the disposition of revenues. The standard approach is to compute deadweight loss, which corresponds to a thought experiment in which the revenues raised by the tax (required by the subsidy) are returned to (obtained from) the individuals as lump-sum payments (levies). In our model, one must specify the manner in which these lump sum transfers are distributed across addictive states. This is important because we do not permit the DM to borrow and lend, thereby redistributing income over time, and consequently over addictive states. A policy that gives the same transfers to all agents regardless of their addictive state would contaminate the experiment by introducing cross-state transfers that, by assumption, are not available to the DM. To eliminate these spurious welfare gains and losses, thereby isolating the effects of taxation and subsidization on usage, one must modify the notion of deadweight loss by requiring that any tax revenue raised from (subsidy paid to) an individual in a given period and state of addiction is redistributed as a lump-sum payment back to (financed by a lump-sum tax on) that individual in the same period and state of addiction. In effect, one visualizes a large population wherein DMs are grouped by addictive state in each period, and all resources affected by taxes, subsidies, and lump-sum transfers remain within these groups. While this construction is artificial, it is necessitated by the artificiality of the no-savings assumption.

First consider policies that tax or subsidize the activities $E, A$, and $R$. Let $\sigma_{s}^{a}$ denote the monetary payment provided to an individual who engages in activity $a$ while in addictive state $s ; \sigma_{s} \equiv\left(\sigma_{s}^{E}, \sigma_{s}^{A}, \sigma_{s}^{R}\right)$, and $\sigma \equiv\left(\sigma_{0}, \ldots, \sigma_{S}\right)$. Note that this class of policies includes subsidized rehabilitation, where the extent of the subsidy may vary with the degree of addiction $\left(\sigma_{s}^{R}>0\right)$. One can also reinterpret the activities $E$ and $A$ so that the class of policies under consideration subsumes needle exchanges, ${ }^{49}$ as well as other "harm reduction" or "Dutch" programs that entail implicit subsidization.

The following result demonstrates that all policies belonging to the class described above are dominated by laissez faire. The intuition is also simple: these policies distort

\footnotetext{
${ }^{49}$ Imagine that action $E$ entails obtaining a clean needle (at some cost $n$ ), while action $A$ does not. Having a dirty needle reduces the likelihood of use if the DM enters the hot cognitive mode $\left(p_{s}^{A}<p_{s}^{E}\right)$. Dirty needles adversely impact health $\left(w_{s}(e, 1, A)<w_{s}(e, 1, E)\right)$. A subsidized needle exchange program involves $\sigma_{s}^{E}>\mathbf{0}$. In this setting, one would not necessarily assume that $u_{s}^{E}>u_{s}^{A}$ (due to the cost $n$ ), but this assumption is not used in the proof of theorem 5 . This depiction of a needle exchange program assumes, of course, that users have opportunities to obtain and store clean needles while in the cold cognitive mode.
} 
behavior in the cold mode, wherein there is no decision-making failure, but have no effect on the hot mode, wherein the DM makes mistakes. As a result, the usual results concerning the inefficiency of taxation and subsidization apply.

Theorem 5: Let $\theta(\sigma)$ denote the vector of parameters derived from $w_{s}(e, x, a)$ when the actions $E, A$, and $R$ are taxed or subsidized according to the schedule $\sigma$ and revenues are distributed as lump sum payments within addictive states. Laissez faire weakly dominates subsidization of rehabilitation: $V_{s}(\theta(\sigma)) \leq V_{s}(\theta(0))$ for all $s$, with strict inequality if $\chi_{s}(\theta(\sigma)) \cap \chi_{s}(\theta(0))=\emptyset$.

We turn our attention next to policies that impose per-unit taxes (or subsidies) on addictive substances. Since usage is excessive in our model (except in the case of constant use), it is natural to conjecture that taxation dominates laissez faire. However, the logic behind theorem 5 should make one suspicious of this conjecture. When the individual chooses to consume in the cold cognitive mode, there is no decision-making failure to correct. When the individual chooses to consume in the hot cognitive mode, but not in the cold mode, there is a decision-making failure. However, as long as choice in the hot mode is insensitive to price, the mistake is not correctable through taxation. A welfare-improving policy corrects mistakes made in the hot cognitive mode without excessively distorting behavior in the cold mode. A tax has exactly the opposite effect: it distorts behavior in the cold mode, and by assumption has no effect on choices made in the hot mode.

Taxation of the addictive substance raises one additional complication. When the DM selects $(E, 0)$ or $(A, 0)$, he pays the tax only in the hot mode, but receives a lump sum payment in both modes. Thus, the tax redistributes income from the hot mode to the cold mode. Since it is unrealistic to assume that the DM has any ability to insure against random realizations of his cognitive mode, it is important to consider the welfare effects of this redistribution. As long as ordinary consumption and the addictive substance are complements, intuition suggests that the redistribution is undesirable and the tax is inefficient; indeed subsidization may be superior to laissez faire. The same intuition suggests that subsidization is inefficient when ordinary consumption and the addictive substance are substitutes; indeed, taxation may be superior to laissez faire. Formally:

Theorem 6: Let $\theta(\tau)$ denote the vector of parameters derived from $w_{s}(e, x, a)$ when the addictive substance is subject to a per-unit tax (or subsidy) $\tau$ and revenues are distributed as lump sum payments within addictive states. Suppose that $w_{s}(e, x, a)$ is concave in $e$.

(i) If $\frac{\partial w_{s}(e, 0, a)}{\partial e} \leq \frac{\partial w_{s}(e, \mathbf{1}, a)}{\partial e}$, then laissez faire dominates a per-unit tax $\tau$ : for every 
state $s$ and $\tau>0, V_{s}(\theta(\tau)) \leq V_{s}(\theta(0))$, with strict inequality if $\chi_{s}(\theta(\tau)) \cap$ $\chi_{s}(\theta(0))=\emptyset$.

(ii) If $\frac{\partial w_{s}(e, 0, a)}{\partial e} \geq \frac{\partial w_{s}(e-q, \mathbf{1}, a)}{\partial e}$ for all e, then laissez faire dominates a per-unit subsidy $\tau$ : for every state $s$ and $\tau<0, V_{s}(\theta(\tau)) \leq V_{s}(\theta(0))$, with strict inequality if $\chi_{s}(\theta(\tau)) \cap \chi_{s}(\theta(0))=\emptyset$.

Notice that the condition in part (ii) requires the degree of substitutability to be sufficiently strong (in light of the fact that reducing income by $q$ tends to increase the marginal benefit from ordinary consumption). Thus the condition in part (i) subsumes the case of separability between ordinary and addictive consumption, whereas the condition in part (ii) does not. With separability, laissez faire dominates a positive tax, but subsidization may dominate laissez faire.

In formulating our model, we made the extreme assumption that use in the hot cognitive mode is completely insensitive to price, while use in the cold mode is potentially price sensitive. As one introduces more price sensitivity in the hot cognitive mode, taxes become more desirable (or less undesirable). However, when price sensitivity is greater in the cold mode than in the hot mode (which seems realistic), the logic of theorem 6 part (i) suggests that substantial taxes on addictive substances are undesirable. 50

\subsection{Criminalization}

Historically, criminalization has been the cornerstone of U.S. drug policy. Criminalization affects users through two distinct channels: a price effect and a scarcity effect. The price effect refers to changes in the marginal cost of using the substance resulting from penalties and other costs imposed on users and suppliers. The scarcity effect refers to interference with the process of matching buyers and sellers: since criminalization forces buyers and sellers to carry out transactions secretively, buyers sometimes have difficulty locating supply. Let $\Delta q$ denote the effective price increase (including the certainty equivalent of penalties on users), and let $\gamma_{s}$ denote the probability that a DM who wants to buy the substance in state $s$ is able to complete the purchase. ${ }^{51}$

\footnotetext{
${ }^{50}$ We conjecture that a small tax would be welfare improving in a model with continuous choices, provided that consumption is sufficiently price elastic in the hot state, irrespective of price sensitivity in the cold state. The logic of this conjecture is as follows. When the tax rate is zero, a small tax creates a second-order welfare loss by distorting behavior in the cold cognitive mode, but introduces a first-order welfare gain by improving choices in the hot mode (since the behavioral response would reduce consumption from an excessive level). Even with a very small demand elasticity in the hot mode, the second effect dominates for sufficiently small tax rates. The welfare effect resulting from redistributions between the hot and cold modes is also first-order; this is why the elasticity in the hot mode must be sufficiently large.

${ }^{51}$ Probabilistic consumption changes the value function somewhat, but the results from section 5 extend to this case. See Goldstein and Kalant [1990] for evidence that drug usage declines as substances
} 
It is instructive to consider the scarcity and price effects separately. Consider first a policy that only generates a price increase. This policy is equivalent to a per-unit-tax policy $(\tau=\Delta q)$ in which the revenue raised by the tax is destroyed. From theorem 6 it follows that the policy is dominated by laissez-faire.

Now consider a policy that generates only scarcity effects. Regardless of the DM's mode, this policy reduces the probability that an agent who wishes to use the substance succeeds in doing so. If the DM chooses $(E, 1)$ in the cold mode, this is detrimental. However, if the DM chooses either $(E, 0)$ or $(A, 0)$ in the cold mode, the effect is beneficial, since it reduces unwanted consumption. Accordingly, the policy reduces welfare when laissez faire leads to constant use, increases welfare when laissez faire leads to non-use, and may either increase or decrease welfare for intermediate cases.

For some parameter values, a beneficial scarcity effect dominates the price effect, and criminalization is superior to laissez faire. This result deserves emphasis, inasmuch as it is difficult to justify a policy of criminalization based on demand-side welfare considerations under many alternative theories of addiction.

Thus far, we have assumed that criminalization has the same scarcity effect on intended and unintended consumption. In practice, an individual who intends to consume an illegal substance can set about locating supply deliberately and systematically, and can maintain stocks in anticipation of transitory difficulties. Consequently, it is natural assume that contrived scarcity leads to a larger reduction in the probability of consuming when the individual does not intend to consume, than when consumption is intentional. In that case, criminalization is more likely to produce a beneficial scarcity effect.

\subsection{Regulated dispensation}

An ideal policy would eliminate consumption only when (i) the DM is in the hot cognitive mode, and (ii) he would have chosen to abstain in the cold mode. Any such policy would achieve the first-best outcome, mimicking the case of a consumer who never suffers from characterization failure ( $p_{s}=0$ for all $s$ ).

The preceding observations lead us to consider a stylized policy of legalization with regulated dispensation. Imagine, in particular, that the government licenses vendors and requires them to respect the following dispensation procedure. Initially, any consumer is permitted to obtain the substance at cost in any period. At the end of any period $t$, a consumer can irreversibly revoke his own eligibility to receive the substance in any future period $t^{\prime}>t$, or set of future periods. We assume that supply is only available through these regulated vendors.

Equipped with this transaction technology, consumers in our model can achieve the

become less available. 
first best outcome. Solving the dynamic programming problem with $p_{s}=0$ for all $s$ yields a deterministic consumption path. The consumer can mimic this outcome by revoking, in period $t$, his eligibility to receive the substance in period $t+1$ if and only if $x=0$ in $t+1$ on the first-best consumption path. In this way, the consumer himself selectively creates optimal scarcity: the substance is available to him only when he would choose to use it while in the cold mode. To put it somewhat differently, this policy allows consumers to make optimal pre-commitments. ${ }^{52}$

Regulated dispensation becomes even more attractive relative to other policies when one introduces heterogeneity across individuals. In our model, usage is optimal for some addicts and suboptimal for others. Since the government cannot distinguish among users with respect to this characteristic, it must impose a common policy for all of them. With regulated dispensation, intentional users can continue to consume the substance without impediment, while unintentional users nevertheless benefit from improved self-control.

In more realistic settings, the simple policy described above would not necessarily permit the consumer to achieve the first-best outcome. If, for example, the desirability of using a substance in any given period depends upon factors (e.g. mood) that remain imperfectly predictable until the period is underway, the individual may in some instances regret revoking his eligibility to obtain the substance. Similarly, if the individual can potentially remain in the hot state for several periods, he may chose to revoke his eligibility to purchase the substance more than one period in advance, and then subsequently regret this decision once the addictive state for the pertinent period is realized. However, under the assumption that the individual never mistakenly elects to revoke future consumption privileges while in the hot mode, the policy always weakly improves welfare ex ante relative to laissez faire. This assumption strikes us as a reasonable approximation. When the use of an addictive substances is pleasurable, it seems unlikely that an individual would, through conditioning, develop a cognitive shortcut wherein the brain is focused on future avoidance of the substance, and therefore inclined to make errors in this direction.

The advantages of regulated dispensation depend upon the government's ability to limit resale of the controlled substance and to suppress alternative sources of supply (black market activity). The approach is more promising in instances where the government can practically restrict administration of the substance to the dispensation centers, thereby impeding the development of an illicit resale market.

\footnotetext{
${ }^{52}$ In a related analysis, Loewenstein, O’Donoghue, and Rabin [2000] emphasize the role of “mandatory waiting periods" in a model where agents systematically overconsume durable goods.
} 


\subsection{Cognitive policies}

In previous theories of addiction, government policy affects use by altering opportunity sets and/or by providing information. Our theory of addiction raises the additional possibility that behavior might also respond to policies that influence cognition. Potential "cognitive policies" fall into two categories: those that alter environmental cues, and those that alter the decision-making biases that emerge when individuals make decisions in the hot mode.

Policies of the first type include restrictions on public consumption, the regulation or prohibition of advertising, and limitations on the location and/or method of sales (e.g. outlawing displays). In each of these cases, the existence of a somewhat unconventional externality (i.e. the impact of one individuals actions on the likelihood that another will make a mistake) potentially justifies public intervention.

Examples of policies within the second category include public advertising campaigns that repeatedly present viscerally charged images concerning the consequences of substance abuse (blackened lungs, impaired brains, gruesome car wrecks, and so forth). By creating new visceral associations between usage and consequences, a campaign may influence the pattern of cognitive activation during the hot mode, and thereby reduce unintended consumption. Moreover, if charged images are presented at the moment of consumption, they may counteract characterization failure by activating thoughts about consequences that the DM would otherwise ignore. It is therefore noteworthy that antismoking campaigns in Canada and Brazil mandate that pack of cigarettes prominently display charged images such as cancerous lungs, severely deformed newborns, and embarrassing situations involving erectile disfunction. Since these are well-known consequences of smoking, the pictures act as emotional reminders, but provide no new information.

The policies discussed in the previous paragraph amount to publicly provided attention management therapy. Since individuals can obtain therapy privately, government intervention is justified only if there is an identifiable market failure, such as free-riding in the provision of non-exclusive (public) broadcast messages.

In the context of our simple stylized model, one can depict the effects of cognitive policies as reductions in the probabilities $p_{s}^{a}$. According to theorem 1, such policies are welfare-improving even though, according the theorem 2, they may lead to greater use and initial experimentation. As with regulated dispensation, cognitive policies are attractive because they are non-coercive, because they accommodate individual heterogeneity, and because they have the potential to reduce unintended use without distorting choice in the cold cognitive mode. 


\section{Conclusions}

We have developed and analyzed a model of addiction based on the premise that cognition processes such as attention affect behavior independently of preferences. In an otherwise standard model of rational addiction, we allow for the possibility that the individual may enter a "hot" cognitive mode in which he always chooses to consume irrespective of underlying preferences (implicitly because inappropriate cognitive shortcuts focus attention on usage and the associated "high"), and we assume that the likelihood of entering this state is related to past choices (implicitly because, through conditioning, previous usage increases the probability of encountering environmental cues which trigger the hot cognitive mode). The individual may also operate in a "cold" cognitive mode, wherein he considers all alternatives and contemplates all consequences, including the effects of current choices on the likelihood of entering the hot cognitive mode in the future. We have argued that the theory is consistent with foundational evidence (e.g. from neuroscience and psychology) concerning the nature of decision-making and addiction. The model is analytically tractable, and it accounts for a broad range of stylized facts concerning addiction. It also generates a plausible qualitative mapping from the characteristics of substances into consumption patterns, thereby providing a basis for empirical tests. Finally, the theory provides a clear standard for evaluating social welfare, and it has a number of striking policy implications.

We have intentionally deferred, until now, an important foundational question: how does one define addiction? Even a casual reading of the literature reveals that this is a difficult and contentious issue. For example, is this phenomenon confined to drugs, or can individuals also be addicted to television, love, and french fries? Our theory suggests an operational definition: an individual is addicted to a substance or activity if he engages in repeated and unwanted consumption. This definition only makes sense in the context of a theory, such as ours, where the notion of unwanted consumption is well-defined. Consumption that is repeated and wanted (as in the case of constant use, which may describe the behavior of regular coffee drinkers), does not constitute addiction. Similarly, an activity that is unwanted but not repeated (an isolated mistake) is not an addiction. Under our definition, addictions may include pathological gambling, overeating, compulsive shopping, and kleptomania. Individuals who suffer from these conditions often experience visceral states comparable to cravings, respond to cues such as stress and advertisements, and exhibit cycles of binges and abstention..$^{53}$

\footnotetext{
${ }^{53}$ See Holden (2001a) for a discussion of recent research concerning the commonalities between these behavioral pathologies and substance addiction. For example, compulsive gamblers and kleptomaniacs respond to drugs such as naltrexone which block the brain's ability to experience euphoric states; compulsive gamblers and bulimics experience sudden relapse even after many years of abstinence.
} 


\section{Appendix}

\section{Proof of Theorem 1}

Step 1: The proof of continuity is standard, and thus omitted.

Step 2: Let $\xi$ be any decision rule such that $\xi_{s} \in \chi_{s}(\theta)$ for all $s$. Consider an increase in $b_{k}^{E}, b_{k}^{A}, u_{k}^{E}, u_{k}^{A}$, or $u_{k}^{R}$, or a decrease in $c_{k}$. Let $\theta^{\prime}$ denote the new vector of parameters. Clearly, if the DM follows $\xi_{s}$ in every state $s$ when the parameters are $\theta^{\prime}$, he will receive an expected discounted payoff in each state $s$ not lower than $V_{s}(\theta)$. Accordingly, the highest achievable expected discounted payoff in each state is not lower than $V_{s}(\theta)$. Since $\chi_{s}\left(\theta^{\prime}\right)$ provides the greatest achievable expected discounted payoff in every state, $V_{s}\left(\theta^{\prime}\right) \geq V_{s}(\theta)$ for all $s$.

Step 3: Let $\xi_{s}$ be any decision rule such that $\xi_{s} \in \chi_{s}(\theta)$ for all $s$. Let $\theta^{\prime}$ denote the vector of parameters obtained by decreasing $p_{k}^{E}$ by $\Delta^{E}$ and $p_{k}^{A}$ by $\Delta^{A}$ (with $\Delta^{a} \geq 0$ ). Consider a probabilistic decision rule $\pi_{s}(a, x)$ (which denotes the probability of selecting $(a, x)$ in state $s)$ defined as follows: (i) for all $s \neq k, \pi_{s}(a, x)=1$ if $\xi_{s}=(a, x)$, and zero otherwise, (2) if $\xi_{k}=(E, 1)$ then $\pi_{k}(E, 1)=1$, (3) if $\xi_{k}=(E, 0)$ then

$\pi_{k}(E, 0)=\frac{1-p_{k}^{E}}{1-p_{k}^{E}+\Delta^{E}}$ and $\pi_{k}(E, 1)=\frac{\Delta^{E}}{1-p_{k}^{E}+\Delta^{E}}$, (4) if $\xi_{k}=(A, 0)$ then $\pi_{k}(A, 0)=$ $\frac{1-p_{k}^{A}}{1-p_{k}^{A}+\Delta^{A}}$ and $\pi_{k}(A, 1)=\frac{\Delta^{A}}{1-p_{k}^{A}+\Delta^{A}}$, and (5) if $\xi_{k}=(R, 0)$, then $\pi_{k}(R, 0)=1$. It is straightforward to verify that following $\pi_{s}(a, x)$ when the parameters are $\theta^{\prime}$ yields an expected discounted payoff in each state $s$ of exactly $V_{s}(\theta)$. Reasoning as in step 2, it follows that $V_{s}\left(\theta^{\prime}\right) \geq V_{s}(\theta)$ for all s. Q.E.D.

\section{Proof of Theorem 2}

We begin the proof with two lemmas.

Lemma 1: Consider $\theta$ and $\theta^{\prime}$ such that: (1) $\theta_{k}^{\prime} \neq \theta_{k}$, (2) $\theta_{i}^{\prime}=\theta_{i}$ for $i \neq k$, and (3) $V_{s}\left(\theta^{\prime}\right) \geq V_{s}(\theta)$ for all s. Then:

(i) for all $j<k, V_{j}\left(\theta^{\prime}\right)-V_{j}(\theta) \leq V_{j+1}\left(\theta^{\prime}\right)-V_{j+1}(\theta)$,

(ii) for all $j>k, V_{j}\left(\theta^{\prime}\right)-V_{j}(\theta) \leq V_{j-1}\left(\theta^{\prime}\right)-V_{j-1}(\theta)$.

Proof: We provide a proof of part (i). The argument for part (ii) is symmetric. In the proof, we make the dependence of $\lambda_{s}^{a, x}$ on $\theta$ explicit by writing $\lambda_{s}^{a, x}(\theta)$.

We claim that for all $j<k, V_{j}\left(\theta^{\prime}\right)-V_{j}(\theta) \leq V_{j+1}\left(\theta^{\prime}\right)-V_{j+1}(\theta)$. Consider first the case $j=1$. (The case $j=0$ is almost identical and thus is omitted). ${ }^{54}$

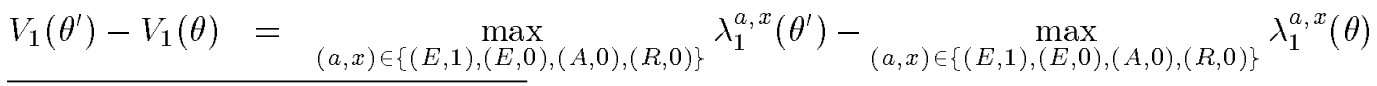

\footnotetext{
${ }^{54}$ The argument makes use of the fact that, for any eight real numbers $z_{1}, \ldots, z_{8}, \max _{i \in\{1,2,3,4\}} z_{i}-$ $\max _{i \in\{5,6,7,8\}} z_{i} \leq \max _{i \in\{1,2,3,4\}}\left\{z_{i}-z_{i+4}\right\}$.
} 


$$
\begin{aligned}
\leq & \max _{(a, x) \in\{(E, \mathbf{1}),(E, 0),(A, 0),(R, 0)\}}\left(\lambda_{1}^{a, x}\left(\theta^{\prime}\right)-\lambda_{1}^{a, x}(\theta)\right) \\
= & \delta \max \left\{V_{2}\left(\theta^{\prime}\right)-V_{2}(\theta),\left(1-p_{1}^{E}\right)\left(V_{1}\left(\theta^{\prime}\right)-V_{1}(\theta)\right)+p_{1}^{E}\left(V_{2}\left(\theta^{\prime}\right)-V_{2}(\theta)\right),\right. \\
& \left.\left(1-p_{1}^{A}\right)\left(V_{1}\left(\theta^{\prime}\right)-V_{1}(\theta)\right)+p_{1}^{A}\left(V_{2}\left(\theta^{\prime}\right)-V_{2}(\theta)\right), V_{1}\left(\theta^{\prime}\right)-V_{1}(\theta)\right\}
\end{aligned}
$$

Consider the last expression. Given the linearity of the second and third terms, there are two possible cases. If the fourth term is the maximand then, since $\delta \in(0,1)$, $V_{1}\left(\theta^{\prime}\right)-V_{1}(\theta)=0 \leq V_{2}\left(\theta^{\prime}\right)-V_{2}(\theta)$ (the last inequality follows from the statement of the theorem). If the first term is the maximand, the claim trivially holds.

Now consider the following induction step. We show that for all $j<k$,

$$
V_{j-1}\left(\theta^{\prime}\right)-V_{j-1}(\theta) \leq V_{j}\left(\theta^{\prime}\right)-V_{j}(\theta) \Rightarrow V_{j}\left(\theta^{\prime}\right)-V_{j}(\theta) \leq V_{j+1}\left(\theta^{\prime}\right)-V_{j+1}(\theta)
$$

Arguing as above, we have

$$
\begin{aligned}
V_{j}\left(\theta^{\prime}\right)-V_{j}(\theta) \leq & \delta \max \left\{V_{j+1}\left(\theta^{\prime}\right)-V_{j+1}(\theta)\right. \\
& \left(1-p_{j}^{E}\right)\left(V_{j-1}\left(\theta^{\prime}\right)-V_{j-1}(\theta)\right)+p_{j}^{E}\left(V_{j+1}\left(\theta^{\prime}\right)-V_{j+1}(\theta)\right) \\
& \left.\left(1-p_{j}^{A}\right)\left(V_{j-1}\left(\theta^{\prime}\right)-V_{j-1}(\theta)\right)+p_{j}^{A}\left(V_{j+1}\left(\theta^{\prime}\right)-V_{j+1}(\theta)\right), V_{j-1}\left(\theta^{\prime}\right)-V_{j-1}(\theta)\right\}
\end{aligned}
$$

As before, there are two possible cases. If the fourth term is the maximand we obtain

$$
V_{j}\left(\theta^{\prime}\right)-V_{j}(\theta) \leq \delta\left(V_{j-1}\left(\theta^{\prime}\right)-V_{j-1}(\theta)\right) \leq \delta\left(V_{j}\left(\theta^{\prime}\right)-V_{j}(\theta)\right)
$$

where the last inequality follows from the induction hypothesis. This implies that $V_{j}\left(\theta^{\prime}\right)-V_{j}(\theta)=0 \leq V_{j+1}\left(\theta^{\prime}\right)-V_{j+1}(\theta)$. If the first term is the maximand, the claim trivially holds. (Note that this establishes the claim only for $j<k$; (5) does not hold for $j=k$ since $\theta_{k}^{\prime} \neq \theta_{k}$ ). Q.E.D.

Lemma 2: Consider $\theta$ and $\theta^{\prime}$ such that: (1) $\theta_{k}^{\prime} \neq \theta_{k}$, (2) $\theta_{i}^{\prime}=\theta_{i}$ for $i \neq k$, and (3) $V_{s}\left(\theta^{\prime}\right) \geq V_{s}(\theta)$ for all s. Then:

(i) For $j<k$, usage in state $j$ is weakly higher with $\theta^{\prime}$ than with $\theta$,

(ii) For $j>k$, usage in state $j$ is weakly lower with $\theta^{\prime}$ than with $\theta$.

Proof: Consider any $\theta$ and $\theta^{\prime}$ that differ only with respect to state $k$, and assume that valuation in all states is higher with $\theta^{\prime}$ than with $\theta$. Lemma 1 tells us that, for $j<k$,

$$
V_{\max \{1, j-1\}}\left(\theta^{\prime}\right)-V_{\max \{1, j-1\}}(\theta) \leq V_{j+1}\left(\theta^{\prime}\right)-V_{j+1}(\theta)
$$

(The case $j=0$ is identical and thus is omitted). Rearranging this expression yields:

$$
\Delta V_{j}\left(\theta^{\prime}\right) \leq \Delta V_{j}(\theta)
$$


Since the parameters for state $j$ have not changed, this implies that usage in state $j$ must be weakly higher with $\theta^{\prime}$ than with $\theta$ (see section 5.1). Using a symmetric argument for $j>k$ completes the proof. Q.E.D.

Now we prove theorem 2. Parts (i) and (ii) follow directly from lemma 2 and theorem 1. The proof of part (iii) proceeds as follows.

Step 1: Consider two parameter vectors, $\underline{\theta}$ and $\bar{\theta}$, such that $\bar{b}_{j}^{E} \geq \underline{b}_{j}^{E}$, with all other components equal. We claim that, if $(E, 1) \in \chi_{j}(\underline{\theta})$, then $(E, 1) \in \chi_{j}(\bar{\theta})$.

Consider any optimal decision function for parameters $\bar{\theta}$ mapping each state to a unique choice, $\bar{\chi}_{s}$ (that is, $\bar{\chi}_{s} \in \chi_{s}(\bar{\theta})$ for all $s$ ). Imagine that the DM follows the optimal decision rule $\bar{\chi}_{s}$, and that he starts from state $j-1$ in period 0 . Let $g_{t}$ indicate the probability of reaching state $j+1$ for the first time in exactly $t$ periods (note that $q_{1}=0$, and that $q_{s}=0$ for all $s$ when $\bar{\chi}_{i}=(R, 0)$ for $\left.i=j, j-1\right)$. Let $G_{t}$ indicate the expected discounted payoff for periods 0 through $t-2$, conditional upon reaching state $j+1$ for the first time in exactly $t$ periods. If there is a positive probability that state $j+1$ will never be reached, let $G_{\infty}$ denote the expected payoff for all periods conditional on this event (otherwise let $G_{\infty}=0$ ). Note that $G_{t}$ and $G_{\infty}$ are the same regardless of whether one evaluates payoffs under $\bar{\theta}$ and $\underline{\theta}$. Note that we can write:

$$
V_{j-1}(\bar{\theta})=\sum_{t=2}^{\infty}\left[\frac{G_{t}}{\delta^{t-1}}+\bar{u}_{j}^{E}+\bar{b}_{j}^{E}+\delta V_{j+\mathbf{1}}(\bar{\theta})\right] g_{t} \delta^{t-1}+\left[1-\sum_{t=2}^{\infty} g_{t}\right] G_{\infty}
$$

Since the DM has the option to follow $\bar{\chi}_{s}$ for $s<j+1$, we know that

$$
V_{j-1}(\underline{\theta}) \geq \sum_{t=2}^{\infty}\left[\frac{G_{t}}{\delta^{t-1}}+\underline{u}_{j}^{E}+\underline{b}_{j}^{E}+\delta V_{j+1}(\underline{\theta})\right] g_{t} \delta^{t-1}+\left[1-\sum_{t=2}^{\infty} g_{t}\right] G_{\infty}
$$

Since $\bar{u}_{j}^{E}=\underline{u}_{j}^{E}$, we have

$$
V_{j-1}(\bar{\theta})-V_{j-1}(\underline{\theta}) \leq \sum_{t=2}^{\infty}\left[\bar{b}_{j}^{E}-\underline{b}_{j}^{E}+\delta\left(V_{j+1}(\bar{\theta})-V_{j+1}(\underline{\theta})\right)\right] g_{t} \delta^{t-1}
$$

Since $\sum_{t=2}^{\infty} g_{t} \delta^{t-1}<1$, this implies

$$
V_{j-1}(\bar{\theta})-V_{j-1}(\underline{\theta}) \leq \bar{b}_{j}^{E}-\underline{b}_{j}^{E}+\delta\left(V_{j+1}(\bar{\theta})-V_{j+1}(\underline{\theta})\right)
$$

Consequently,

$$
\begin{aligned}
\Delta V_{j}(\bar{\theta})-\Delta V_{j}(\underline{\theta}) & =\left[V_{j-1}(\bar{\theta})-V_{j+1}(\bar{\theta})\right]-\left[V_{j-1}(\underline{\theta})-V_{j+1}(\underline{\theta})\right] \\
& =\left[V_{j-1}(\bar{\theta})-V_{j-1}(\underline{\theta})\right]-\left[V_{j+1}(\bar{\theta})-V_{j+1}(\underline{\theta})\right] \\
& \leq \bar{b}_{j}^{E}-\underline{b}_{j}^{E}-(1-\delta)\left(V_{j+1}(\bar{\theta})-V_{j+1}(\underline{\theta})\right) \\
& \leq \frac{\bar{b}_{j}^{E}-\underline{b}_{j}^{E}}{\delta}
\end{aligned}
$$


(where, in the last step, we have used $\delta<1$ along with the fact that, by theorem 1 , $\left.V_{j+1}(\bar{\theta}) \geq V_{j+1}(\underline{\theta})\right)$. Since, by assumption, $(E, 1) \in \chi_{j}(\underline{\theta})$, we know that $\Delta V_{j}(\underline{\theta}) \leq \frac{\underline{b}_{j}^{E}}{\delta}$. Consequently, we have

$$
\begin{aligned}
\Delta V_{j}(\bar{\theta}) & \leq \Delta V_{j}(\underline{\theta})+\frac{\bar{b}_{j}^{E}-\underline{b}_{j}^{E}}{\delta} \\
& \leq \frac{\underline{b}_{j}^{E}}{\delta}+\frac{\bar{b}_{j}^{E}-\underline{b}_{j}^{E}}{\delta}=\frac{\bar{b}_{j}^{E}}{\delta}
\end{aligned}
$$

But this implies $(E, 1) \in \chi_{j}(\bar{\theta})$.

Step 2: Consider any $\bar{\theta}$ and $\underline{\theta}$ with $\bar{b}_{j}^{E} \geq \underline{b}_{j}^{E}$ and/or $\bar{p}_{j}^{E} \leq \underline{p}_{j}^{E}$ (and all other components equal). We claim that, if $(E, x) \notin \chi_{j}(\bar{\theta})$ for $x=0,1$, then $\chi_{j}(\underline{\theta})=\chi_{j}(\bar{\theta})$. It is easy to check that $V_{s}(\underline{\theta})=V_{s}(\bar{\theta})$ satisfies (1) through (4) for all $s$ when $\theta=\underline{\theta}$. Consequently, $V_{s}(\bar{\theta})$ is also the optimized value function with $\underline{\theta}$, and the elements of $\chi_{j}(\bar{\theta})$ maximize the expression (4) for state $j$ with $\theta=\underline{\theta}$.

Step 3: Consider any $\bar{\theta}$ and $\underline{\theta}$ with $\bar{b}_{j}^{E} \geq \underline{b}_{j}^{E}$ and/or $\bar{p}_{j}^{E} \leq \underline{p}_{j}^{E}$ (and all other components equal). We claim that, if $(E, 0) \in \chi_{j}(\underline{\theta})$ for $x=0,1$, then $(a, 0) \notin \chi_{j}(\bar{\theta})$ for $a=A, R$. Suppose on the contrary that $(a, 0) \in \chi_{j}(\bar{\theta})$ for $a \in\{A, R\}$. Then, arguing as in step $2, V_{s}(\underline{\theta})=V_{s}(\bar{\theta})$. It is easy to check that this implies $(E, 0)$ cannot maximize the expression (4) for state $j$ with $\theta=\underline{\theta}$, so $(E, 0) \notin \chi_{j}(\underline{\theta})$, which is a contradiction.

Combining steps 1,2 , and 3 , we conclude that, for $\bar{\theta}$ and $\underline{\theta}$ with $\bar{b}_{j}^{E} \geq \underline{b}_{j}^{E}$ (and all other components equal), any element of $\chi_{j}(\underline{\theta}) \backslash \chi_{j}(\bar{\theta})$ must involve less usage than any element of $\chi_{j}(\bar{\theta})$, and any element of $\chi_{j}(\bar{\theta}) \backslash \chi_{j}(\underline{\theta})$ must involve more usage than any element of $\chi_{j}(\underline{\theta})$. Consequently, usage is (weakly) increasing in $b_{j}^{E}$.

Step 4: Consider any $\bar{\theta}$ and $\underline{\theta}$ that differ only with respect to $p_{j}^{E}, u_{j}^{R}$, and $/$ or $c_{j}$. We claim that, if $(E, 1) \in \chi_{j}(\bar{\theta})$, then $(E, 1) \in \chi_{j}(\underline{\theta})$. This is a corollary of Theorem 3 , part (i), which is proven below.

Combining steps 2, 3, and 4, we conclude that, for $\bar{\theta}$ and $\underline{\theta}$ with $\bar{p}_{j}^{E} \leq \underline{p}_{j}^{E}$ (and all other components equal), any element of $\chi_{j}(\underline{\theta}) \backslash \chi_{j}(\bar{\theta})$ must involve less usage than any element of $\chi_{j}(\bar{\theta})$, and any element of $\chi_{j}(\bar{\theta}) \backslash \chi_{j}(\underline{\theta})$ must involve more usage than any element of $\chi_{j}(\underline{\theta})$. Consequently, usage is (weakly) decreasing in $p_{j}^{E}$.

Step 5: Consider any $\bar{\theta}$ and $\underline{\theta}$ with $\underline{u}_{j}^{R} \geq \bar{u}_{j}^{R}$ and/or $\underline{c}_{j} \leq \bar{c}_{j}$ (and all other components equal). We claim that if $(R, 0) \notin \chi_{j}(\underline{\theta})$, then $\chi_{j}(\bar{\theta})=\chi_{j}(\underline{\theta})$. The argument is analogous to that given in step 2.

Step 6: Consider any $\bar{\theta}$ and $\underline{\theta}$ with $\underline{u}_{j}^{R} \geq \bar{u}_{j}^{R}$ and/or $\underline{c}_{j} \leq \bar{c}_{j}$ (and all other components equal). We claim that if $(R, 0) \in \chi_{j}(\bar{\theta})$, then $(a, 0) \notin \chi_{j}(\underline{\theta})$ for $a=E, A$. The argument is analogous to that given for step 3 .

Combining steps 4,5 , and 6 , we conclude that, for $\bar{\theta}$ and $\underline{\theta}$ with $\underline{u}_{j}^{R} \geq \bar{u}_{j}^{R}$ and /or $\underline{c}_{j} \leq \bar{c}_{j}$ (and all other components equal), any element of $\chi_{j}(\underline{\theta}) \backslash \chi_{j}(\bar{\theta})$ must involve less 
usage than any element of $\chi_{j}(\bar{\theta})$, and any element of $\chi_{j}(\bar{\theta}) \backslash \chi_{j}(\underline{\theta})$ must involve more usage than any element of $\chi_{j}(\underline{\theta})$. Consequently, usage is (weakly) decreasing in $u_{j}^{R}$ and (weakly) increasing in $c_{j}$. Q.E.D.

\section{Proof of Theorem 3}

(i) Consider some $\theta^{\prime}$ for which $(E, 1) \in \chi_{j}\left(\theta^{\prime}\right)$. Suppose that $\theta^{\prime \prime}$ coincides with $\theta^{\prime}$ except for $p_{j}^{E}, p_{j}^{A}, u_{j}^{A}, u_{j}^{R}, b_{j}^{A}$, and/or $c_{j}$ (subject to the restrictions imposed by assumptions 1 and 2). We claim that $(E, 1) \in \chi_{j}\left(\theta^{\prime \prime}\right)$. By construction, $V_{s}\left(\theta^{\prime}\right)$ satisfies (1) through (4) for $\theta=\theta^{\prime}$. We argue that $V_{s}\left(\theta^{\prime \prime}\right)=V_{s}\left(\theta^{\prime}\right)$ also satisfies (1) through (4) for $\theta=\theta^{\prime \prime}$. Under the hypothesis that $V_{s}\left(\theta^{\prime \prime}\right)=V_{s}\left(\theta^{\prime}\right)$, we have $\lambda_{s}^{a, x}\left(\theta^{\prime}\right)=\lambda_{s}^{a, x}\left(\theta^{\prime \prime}\right)$ for all $s \neq j$, and for $(s, a, x)=(j, E, 1)$. Thus, (4) is satisfied for all $s \neq j$. Since $(E, 1) \in \chi_{j}\left(\theta^{\prime}\right)$ we know that $(E, 1)$ maximizes (4) for state $j$ with $\theta=\theta^{\prime}$, which is equivalent to $\Delta V_{j}\left(\theta^{\prime}\right) \leq \frac{b_{j}^{E}}{\delta}$. But then, under our hypothesis, $\Delta V_{j}\left(\theta^{\prime \prime}\right) \leq \frac{b_{j}^{E}}{\delta}$ as well, so $(E, 1)$ remains a maximizer for state $j$ with $\theta=\theta^{\prime \prime}$. Since $\lambda_{s}^{E, 1}\left(\theta^{\prime}\right)=\lambda_{s}^{E, 1}\left(\theta^{\prime \prime}\right)$, the maximized value of (4) is unchanged with $\theta=\theta^{\prime \prime}$. Accordingly, $V_{s}\left(\theta^{\prime \prime}\right)=V_{s}\left(\theta^{\prime}\right)$ is the maximized value function when $\theta=\theta^{\prime \prime}$, and $\chi_{j}\left(\theta^{\prime \prime}\right)$ is the set of optimal choices in state $j$.

(ii) Consider some $\theta$ for which $(R, 0) \in \chi_{n}(\theta)$. Construct some $\theta^{\prime}$ by increasing the values of $p_{k}^{a}$ or $c_{k}$, or by decreasing the values of $b_{k}^{a}$ or $u_{k}^{a}$, for some $k>n$. By theorem 2 part (i), $(R, 0) \in \chi_{n}(\theta)$. Consider the optimization problem formed by restricting the model to addictive states 0 through $n$, and imposing the action $(R, 0)$ in state $n$. This problem has the same solution as the complete problems with either $\theta$ or $\theta^{\prime}$ on states 0 through $n$ since conditions (1) to (4) coincide on these states. It follows that the optimal solution at $\theta^{\prime}$ remains unchanged for states 0 through $n$.

(iii) The proof is analogous to that of part (ii). Q.E.D.

\section{Proof of Theorem 4}

Step 1. We show that $V_{0}(\theta) \geq V_{1}(\theta)$. Since $(E, 1)$ is an option at $s=0$, we have that $V_{0}(\theta) \geq u_{0}^{E}+b_{0}^{E}+\delta V_{1}(\theta)$. Furthermore, since the substance is destructively addictive, we must have that $V_{1}(\theta) \leq \frac{u_{1}^{E}+b_{1}^{E}}{1-\delta} \leq \frac{u_{0}^{E}+b_{0}^{E}}{1-\delta}$. These two inequalities imply that $V_{0}(\theta) \geq(1-\delta) V_{1}(\theta)+\delta V_{1}(\theta)=V_{1}(\theta)$.

Step 2 Now we show that $V_{s-1}(\theta) \geq V_{s}(\theta)$ implies $V_{s}(\theta) \geq V_{s+1}(\theta)$. We consider four cases. These cases are not mutually exclusive, but they are exhaustive.

(i) $(E, 1) \in \chi_{s}(\theta)$. In this case, $V_{s}(\theta)=u_{s}^{E}+b_{s}^{E}+\delta V_{s+1}(\theta)$. Furthermore, since states lower than $s$ are never reached (once the DM is in state $s$ ), and the substance is destructively addictive, we have that $V_{s+1}(\theta) \leq \frac{u_{s}^{E}+b_{s}^{E}}{1-\delta}$. The argument then proceeds as in step 1.

(ii) $(R, 0) \in \chi_{s+1}(\theta)$. In this case we have that

$$
V_{s+1}(\theta)=u_{s+\mathbf{1}}^{R}-c_{s+\mathbf{1}}+\delta V_{s}(\theta) \leq u_{s}^{E}+b_{s}^{E}+\delta V_{s}(\theta) .
$$


Also, $V_{s}(\theta) \geq u_{s}^{E}+b_{s}^{E}+\delta V_{s+\mathbf{1}}(\theta)$ since $(E, 1)$ is an option at state $s$. This implies that $V_{s}(\theta) \geq V_{s+1}(\theta)-\delta V_{s}(\theta)+\delta V_{s+1}(\theta)$ and thus $V_{s}(\theta) \geq V_{s+1}(\theta)$.

(iii) $(E, 1) \in \chi_{s+1}(\theta)$. In this case $V_{s+1}(\theta) \leq \frac{u_{s+1}^{E}+b_{s+1}^{E}}{1-\delta} \leq \frac{u_{s}^{E}+b_{s}^{E}}{1-\delta}$, so $u_{s}^{E}+b_{s}^{E} \geq$ $(1-\delta) V_{s+1}(\theta)$. Also, at state $s$ we have that $V_{s}(\theta) \geq u_{s}^{E}+b_{s}^{E}+\delta V_{s+1}(\theta)$ (since $(E, 1)$ is available as an option). Combining these inequalities yields $V_{s}(\theta) \geq V_{s+1}(\theta)$.

(iv) Two conditions hold: (a) either $(E, 0) \in \chi_{s+1}(\theta)$ or $(A, 0) \in \chi_{s+1}(\theta)$, and (b) $(E, 1) \notin \chi_{s}(\theta)$. Consider $a$ such that $(a, 0) \in \chi_{s+1}(\theta)$. Since $b_{s+1}^{E} \geq 0$, we must have $V_{s}(\theta) \geq V_{s+2}(\theta)$ (otherwise $(E, 1)$ would be preferable to $(a, 0)$ at $s+1$ ). Suppose, towards a contradiction, that $V_{s+1}(\theta)>V_{s}(\theta)$. Then $V_{s+1}(\theta)>V_{s+2}(\theta)$. Then we have that

$$
\begin{aligned}
V_{s+1}(\theta) & =p_{s+1}^{a}\left(u_{s+1}^{a}+b_{s+1}^{a}+\delta V_{s+2}(\theta)\right)+\left(1-p_{s+1}^{a}\right)\left(u_{s+1}^{a}+\delta V_{s}(\theta)\right) \\
& \leq p_{s+1}^{a}\left(u_{s+1}^{a}+b_{s+1}^{a}+\delta V_{s+1}(\theta)\right)+\left(1-p_{s+1}^{a}\right)\left(u_{s+1}^{a}+\delta V_{s-1}(\theta)\right) \\
& \leq p_{s+1}^{a}\left(u_{s}^{a}+b_{s}^{a}+\delta V_{s+1}(\theta)\right)+\left(1-p_{s+1}^{a}\right)\left(u_{s}^{a}+\delta V_{s-1}(\theta)\right) \\
& \leq p_{s}^{a}\left(u_{s}^{a}+b_{s}^{a}+\delta V_{s+1}(\theta)\right)+\left(1-p_{s}^{a}\right)\left(u_{s}^{a}+\delta V_{s-1}(\theta)\right) \text { (see below) } \\
& \leq V_{s}(\theta)
\end{aligned}
$$

which is a contradiction. The third inequality follows because $(E, 1) \notin \chi_{s}(\theta)$, which requires $\Delta V_{s}(\theta) \geq \frac{b_{s}^{E}}{\delta}$, which in turn implies $u_{s}^{a}+\delta V_{s-1}(\theta) \geq u_{s}^{a}+b_{s}^{a}+\delta V_{s+1}(\theta)$.

Step 3 At $s=0$ the DM can guarantee himself a constant payoff $\frac{u_{o}^{A}}{1-\delta}$, which implies that $\Delta V_{0}(\theta) \geq 0$. By the conditions in section 5.1, it follows that $(E, 1) \notin \chi_{0}(\theta)$ when $b_{0}^{E}<0$. Q.E.D.

\section{Proof of Theorem 5}

Let $T_{s}(\sigma)$ denote the lump-sum tax (possibly negative) that the DM pays in state $s$ with the tax-subsidy scheme $\sigma$. We claim that, if the DM follows $\chi_{s}(\theta(\sigma))$ when the parameter vector is $\theta(0)$, he achieves an expected discounted payoff in each state $s$ of $V_{s}(\theta(\sigma))$. From this it follows immediately that the optimized value function with $\theta(0)$ satisfies $V_{s}(\theta(0)) \geq V_{s}(\theta(\sigma))$, as required, with strict inequality if no member of $\chi_{s}(\theta(\sigma))$ is optimal with the parameter vector $\theta(0)$.

To establish the claim, suppose that the DM selects a decision rule $(\zeta, \psi)$ with $\left(\zeta_{s}, \psi_{s}\right) \in \chi_{s}(\theta(\sigma))$ for all $s$. Government budget balance (with no redistributions between addictive states) requires $T_{s}(\sigma)=\sigma_{s}^{\zeta_{s}}$. If $\zeta_{s}=E$ or $A$, then with tax-subsidy scheme $\sigma$ the choice $\left(\zeta_{s}, \psi_{s}\right)$ confers instantaneous utility in state $s$ of

$$
\begin{aligned}
u_{s}^{\zeta_{s}}(\sigma)+\psi_{s} b_{s}^{\zeta_{s}}(\sigma) & =w_{s}\left(y_{s}-\psi_{s} q-T_{s}(\sigma)+\sigma_{s}^{\zeta_{s}}, \psi_{s}, \zeta_{s}\right) \\
& =w_{s}\left(y_{s}-\psi_{s} q, \psi_{s}, \zeta_{s}\right) \\
& =u_{s}^{\zeta_{s}}(0)+\psi_{s} b_{s}^{\zeta_{s}}(0)
\end{aligned}
$$


Similarly, if $\zeta_{s}=R$, then with tax-subsidy scheme $\sigma$ the choice $\left(\zeta_{s}, \psi_{s}\right)$ confers instantaneous utility in state $s$ of

$$
\begin{aligned}
u_{s}^{R}(\sigma)-c_{s}(\sigma) & =w_{s}\left(y_{s}-T_{s}(\sigma)-r_{s}+\sigma_{s}^{R}, 0, R\right) \\
& =w_{s}\left(y_{s}-r_{s}, 0, R\right) \\
& =u_{s}^{R}(0)-c_{s}(0)
\end{aligned}
$$

It follows that the decision rule $(\zeta, \psi)$ generates the same expected discounted utility in all states with $\sigma$ as with no tax-subsidy scheme. Q.E.D.

\section{Proof of Theorem 6}

Let $T_{s}(\tau)$ denote the lump-sum payment (possibly negative) that the DM pays in state $s$ with the tax-subsidy $\tau$. We claim that, if the DM follows $\chi_{s}(\theta(\tau))$ when the parameter vector is $\theta(0)$, he achieves an expected discounted payoff in each state $s$ of no less than $V_{s}(\theta(\tau))$. From this it follows immediately that the optimized value function with $\theta(0)$ satisfied $V_{s}(\theta(0)) \geq V_{s}(\theta(\tau))$, as required, with strict inequality if no member of $\chi_{s}(\theta(\sigma))$ is optimal with parameter vector $\theta(0)$.

To establish the claim, suppose that the DM selects a decision rule $(\zeta, \psi)$ with $\left(\zeta_{s}, \psi_{s}\right) \in \chi_{s}(\theta(\tau))$ for all $s$. Government budget balance (with no redistributions between addictive states) requires $T_{s}(\sigma)=\left(\psi_{s}+\left(1-\psi_{s}\right) p_{s}^{\zeta_{s}}\right) \tau$ when $\zeta_{s}=E$ or $A$, and $T_{s}(\sigma)=0$ when $\zeta_{s}=R$.

If $\left(\zeta_{s}, \psi_{s}\right)=(E, 1)$, then with tax-subsidy $\tau$ the choice $(E, 1)$ confers instantaneous utility in state $s$ of

$$
\begin{aligned}
u_{s}^{E}(\tau)+b_{s}^{E}(\tau) & =w_{s}\left(y_{s}-(q+\tau)+T_{s}(\tau), 1, E\right) \\
& =w_{s}\left(y_{s}-q, 1, E\right) \\
& =u_{s}^{E}(0)+b_{s}^{E}(0)
\end{aligned}
$$

If $\left(\zeta_{s}, \psi_{s}\right)=(R, 0)$, then with tax-subsidy $\tau$ the choice $(R, 0)$ confers instantaneous utility in state $s$ of

$$
\begin{aligned}
u_{s}^{E}(\tau)-c_{s}(\sigma) & =w_{s}\left(y_{s}-r_{s}+T_{s}(\tau), 0, E\right) \\
& =w_{s}\left(y_{s}-r_{s}, 0, E\right) \\
& =u_{s}^{R}(0)-c_{s}(0)
\end{aligned}
$$

If $\left(\zeta_{s}, \psi_{s}\right)=(a, 0)$ with $a=E$ or $A$, then with tax-subsidy $\tau$ the choice $(a, 0)$ confers instantaneous utility in state $s$ of

$$
u_{s}^{a}(\tau)+p_{s}^{a} b_{s}^{a}(\tau)=p_{s}^{a} w_{s}\left(y_{s}-(q+\tau)+T_{s}(\tau), 1, a\right)+\left(1-p_{s}^{a}\right) w_{s}\left(y_{s}+T_{s}(\tau), 0, a\right)
$$




$$
\begin{aligned}
& =p_{s}^{a} w_{s}\left(y_{s}-(q+\tau)+p_{s}^{a} \tau, 1, a\right)+\left(1-p_{s}^{a}\right) w_{s}\left(y_{s}+p_{s}^{a} \tau, 0, a\right) \\
& =p_{s}^{a} w_{s}\left(y_{s}-q, 1, a\right)+\left(1-p_{s}^{a}\right) w_{s}\left(y_{s}, 0, a\right)+\Omega \\
& =u_{s}^{a}(0)+p_{s}^{a} b_{s}^{a}(0)+\Omega
\end{aligned}
$$

where

$$
\Omega=\left(1-p_{s}^{a}\right) \int_{0}^{p_{s}^{a} \tau} \frac{\partial w_{s}\left(y_{s}+z, 0, a\right)}{\partial e} d z-p_{s}^{a} \int_{-\left(1-p_{s}^{a}\right) \tau}^{0} \frac{\partial w_{s}\left(y_{s}-q+z, 1, a\right)}{\partial e} d z
$$

Now suppose that $\frac{\partial w_{s}(e, 0, a)}{\partial e} \leq \frac{\partial w_{s}(e, \mathbf{1}, a)}{\partial e}$ (as in part (i) of the theorem). Imagine that $\tau>0$. Since $w_{s}(e, x, a)$ is concave in $e$,

$$
\begin{aligned}
\Omega & \leq\left(1-p_{s}^{a}\right) \int_{0}^{p_{s}^{a} \tau} \frac{\partial w_{s}\left(y_{s}+z, 1, a\right)}{\partial e} d z-p_{s}^{a} \int_{-\left(1-p_{s}^{a}\right) \tau}^{0} \frac{\partial w_{s}\left(y_{s}-q+z, 1, a\right)}{\partial e} d z \\
& \leq\left(1-p_{s}^{a}\right) \int_{0}^{p_{s}^{a} \tau} \frac{\partial w_{s}\left(y_{s}-q+z, 1, a\right)}{\partial e} d z-p_{s}^{a} \int_{-\left(1-p_{s}^{a}\right) \tau}^{0} \frac{\partial w_{s}\left(y_{s}-q+z, 1, a\right)}{\partial e} d z \\
& =\left(1-p_{s}^{a}\right) w_{s}\left(y_{s}-q+p_{s}^{a} \tau, 1, a\right)+p_{s}^{a} w_{s}\left(y_{s}-q-\left(1-p_{s}^{a}\right) \tau, 1, a\right)-w_{s}\left(y_{s}-q, 1, a\right) \\
& \leq 0,
\end{aligned}
$$

where the last inequality uses concavity and the fact that $\left(1-p_{s}^{a}\right)\left(y_{s}-q+p_{s}^{a} \tau\right)+p_{s}^{a}\left(y_{s}-\right.$ $\left.q-\left(1-p_{s}^{a}\right) \tau\right)=y_{s}-q$. Thus, for all states, the choice $\left(\zeta_{s}, \psi_{s}\right)$ confers weakly greater instantaneous utility with no tax than with the tax. It follows that the decision rule $(\zeta, \psi)$ generates weakly greater expected discounted utility in all states with no tax than with $\tau>0$.

Finally, suppose that $\frac{\partial w_{s}(e, 0, a)}{\partial e} \geq \frac{\partial w_{s}(e-q, \mathbf{1}, a)}{\partial e}$ (as in part (ii) of the theorem). Imagine that $\tau<0$. Then

$$
\begin{aligned}
\Omega & \leq p_{s}^{a} \int_{0}^{-\left(1-p_{s}^{a}\right) \tau} \frac{\partial w_{s}\left(y_{s}+z, 0, a\right)}{\partial e} d z-\left(1-p_{s}^{a}\right) \int_{p_{s}^{a} \tau}^{0} \frac{\partial w_{s}\left(y_{s}+z, 0, a\right)}{\partial e} d z \\
& =p_{s}^{a} w_{s}\left(y_{s}-q-\left(1-p_{s}^{a}\right) \tau, 1, a\right)+\left(1-p_{s}^{a}\right) w_{s}\left(y_{s}-q+p_{s}^{a} \tau, 1, a\right)-w_{s}\left(y_{s}-q, 1, a\right) \\
& \leq 0
\end{aligned}
$$

As before, it follows that the decision rule $(\zeta, \psi)$ generates weakly greater expected discounted utility in all states with no subsidy than with $\tau<0$. Q.E.D. 


\section{References}

[1] Abratt, R. and S. Goodey (1990) "Unplanned Buying and In-Store Stimuli in Supermarkets," Managerial and Decision Economics," 111-21.

[2] Barkow, Jerome, Leda Cosmides, and John Tooby (1995) The Adapted Mind: Evolutionary Psychology and the Generation of Culture. Oxford: Oxford University Press.

[3] Becerra, Lino, Hans Breiter, Roy Wise, Gilberto Gonzalez, and David Borsook (2001) "Reward Circuitry and Activation by Noxious Thermal Stimuli," Neuron, 32:927-46.

[4] Bechara, A., A. Damasio, H. Damasio, and S. Anderson (1994) "Insensitivity to Future Consequences Following Damage to Human Prefrontal Cortex," Cognition, $7-15$.

[5] Bechara, A., D. Tranel, A. Damasio, and H. Damasio (1996) "Failure to Respond Autonomically to Anticipated Future Outcomes Following Damage to the Prefrontal Cortex," Cerebral Cortex, 215-25.

[6] Bechara, A., D. Tranel, A. Damasio, and H. Damasio (1997) "Deciding Advantegeously Before Knowing the Advantageous Strategy," Science, 1293-95.

[7] Becker, Gary and Kevin Murphy (1988) "A Theory of Rational Addiction," Journal of Political Economy, 96(4), 675-700.

[8] Berke, J.D. and S. E. Hyman (2000) "Addiction, Dopamine, and the Molecular Mechanisms of Memory," Neuron, 25: 515-32.

[9] Bernheim, Douglas and Antonio Rangel (2002) "Cognition, Evaluation, and Hedonics: A Brain Processes Model of Decision-Making", Stanford manuscript.

[10] Berridge, K. (1996) "Food Reward: Brain Substrates of Wanting and Liking," Neuroscience and Biobehavioral Reviews, 20(1):1-25

[11] Berridge, K. (1999) "Pleasure, Pain, Desire, and Dread: Hidden Core Processes of Emotion," in Well-Being: The Foundations of Hedonic Pscyhology, D. Kaheman, E. Diener, and N. Schwarz (eds.), 525-57

[12] Berridge, Kent, and Terry Robinson (1998) "What is the role of dopamine in reward: hedonic impact, reward learning, or incentive salience?", Brain Research Review, 28:309-69.

[13] Breiter, Hans C., et. al. (1997) "Acute Effects of Cocaine on Human Brain Activity and Emotion," Neuron, 19:591-611. 
[14] Center for Disease Control (1993), "Smoking-Attributable Mortality and Years of Potential Life Lost - United States, 1990," Morbidity and Mortality Weekly Report, $42(33), 645-8$.

[15] Chaloupka, F. and K. Warner (2001) "The Economics of Smoking", in J. Newhouse and D. Cutler (eds.), Handbook of Health Economics, North-Holland.

[16] Damasio, Antonio (1994) Descartes Error: Emotion, Reason, and the Human Brain. New York: Putnam.

[17] Davis, M. (1992a) "The Role of the Amygdala in Fear and Anxiety," Annual Review of Neuroscience, 353-75.

[18] Davis, M. (1992b) "The Role of the Amygdala in Conditioned Fear," In J.P. Aggleton (ed.), The Amygdala: Neurobiological Aspects of Emotion, Memory, and Mental Disfunction, New York: Wiley-Liss.

[19] Gardner, Eliot and James David (1999) "The Neurobiology of Chemical Addiction," in Getting Hooked: Rationality and Addiction, edited by Jon Elster and Ole-Jorgen Skog. Cambridge: Cambridge University Press.

[20] Gawin, F.H. (1991) "Cocaine addiction: Psychology and neurophysiology," Science, $1580-6$.

[21] Gazzaniga, M. (1990). Transcript of interview published in the National Review.

[22] Gazzaniga, M. (1994). Transcript of interview published in the National Review.

[23] Gerstein, Dean, et. al. (1999). Gambling Impact and Behavior Study, Report to the National Gambling Impact Study Commission. Chicago, IL: National Opinion Research Center.

[24] Goldstein, A. (2001) Addiction: From Biology to Drug Policy. Second Edition. New York: Oxford University Press.

[25] Goldstein, A. and H. Kalant (1990) "Drug Policy: Striking the Right Balance," Science 249: 1513-21.

[26] Gruber, Jonathan, and Botond Koszegi (2001) "Is Addiction "Rational"? Theory and Evidence," Quarterly Journal of Economics, 116(4): 1261-1303 .

[27] Gul, Faruk and Wolfgang Psendorfer (2001a), "Temptation and Self-Control", Econometrica 69(6): 1403-35.

[28] Gul, Faruk and Wolfgang Psendorfer (2001b) "A Theory of Addiction," Princeton mimeo. 
[29] Harris, J.E. (1993) Deadly Choices: Coping with Health Risks in Everyday Life. New York: Basic Books.

[30] Helmuth, L. (2001) "Beyond the Pleasure Principle," Science, 294:983-84.

[31] Holden, C. (2001a) “'Behavioral' addictions: Do they exist?” Science, 294:980-82.

[32] Holden, C. (2001b) "Zapping Memory Centers Triggers Drug Craving," Science, 292:1039

[33] Hser, Y.I., D. Anglin, and K. Powers (1993) "A 24-year follow-up study of California narcotics addicts," Archives of General Psychiatry, 50:577-84.

[34] Hser, Y.I., V. Hoffman, C. Grella, and M.D. Anglin (2001) "A 33 year follow-up of narcotics addicts," Archives of General Psychiatry, 58:503-8.

[35] Hung, Angela (2000) “A Behavioral Theory of Addiction," Caltech, manuscript.

[36] Janis, I. (1967) "Effects of fear arousal on attitudinal change," Advances in Experimental Psychology, 167-224.

[37] Hyman, Steven and Robert Malenka [2001] "Addiction and the Brain: The Neurobiology of Compulsion and Its Persistence," Nature Reviews: Neurosience, 2: 695-703.

[38] Koob, G. and M. LeMoal (1997) "Drug Abuse: Hedonic Homeostatic Dysregulation", Science 278, October, 52-8.

[39] Laibson, David (1997) "Golden Eggs and Hyperbolic Discounting," Quarterly Journal of Economics, (112):443-77.

[40] Laibson, David (2001) "A Cue-Theory of Consumption," Quarterly Journal of Economics, 116(1): 81-120.

[41] LeDoux, Joseph (1992) "Emotion and the Amygdala." In J.P. Aggleton (ed.) The Amygdala: Neurobiological Aspects of Emotion, Memory and Mental Dysfunction, pp. 339-51. New York: Wiley-Liss.

[42] LeDoux, Joseph (1993) "Emotional Networks in the Brain," In Michael Davis and Jeannette Haviland (eds.), Handbook of Emotions, New York: The Guildford Press.

[43] LeDoux, Joseph (1998) The Emotional Brain: The Mysterious Underpinnings of Emotional Life. Touchstone books.

[44] Loewenstein, George (1996) "Out of Control: Visceral Influences on Behavior," Organizational Behavior and Human Decision Processes, 65(3) 272-92. 
[45] Loewenstein, George (1999) “A Visceral Account of Addiction," in Jon Elster and Ole-Jorgen Skog (eds.) Rationality and Addiction. Cambridge: Cambridge University Press.

[46] Loewenstein, George, and Jennifer S. Lerner (2001) "The Role of Affect in Decision Making," in R.J. Davidson, H.H. Goldsmith, and K.R. Scherer (eds.) Handbook of Affective Science. Oxford: Oxford University Press.

[47] Loewenstein, George, Ted O'Donoghue, and Matthew Rabin (2000) "Projection Bias in Predicting Future Utility," mimeo.

[48] Massing, Michael. The Fix. Los Angeles: University of California Press, 2000.

[49] MacCoun, R. and P. Reuter (2001) Drug War Heresies: Learning from Other Vices, Times, and Places. Cambridge University Press.

[50] Metcalfe, Janet, and Walter Mischel (1999) “A Hot/Cool-System Analysis of Delay of Gratification: Dynamics of Willpower," Psychological Review, 106(1):3-19.

[51] Miron, J. and J. Zwiebel (1995) "The Economic Case Against Drug Prohibition," Journal of Economic Perspectives, 9(4):175-92.

[52] Mischel, W. (1974) "Processes in delay of gratification," In Advances in Experimental Social Psychology, volume 7, D. Berkowitz editor, 249-272.

[53] Mischel, W. and B. Moore (1973) "Cognitive Appraisals and Transformations in the Delay of Gratification," Journal of Personality and Social Psychology, 28:172-9.

[54] Michel, W., Y. Shoda, and M. Rodriguez (1992) "Delay of Gratification in Children," in Choice over Time, G. Loewenstein and Jon Elster editors, New York: Rusell Sage.

[55] National Institute on Alcohol Abuse and Alcoholism (2001), "Economic Perspectives in Alcholism Research", Alcohol Alert, National Institutes of Health, No. 51.

[56] National Institute on Drug Abuse (1998). The Economic Costs of Alcohol and Drug Abuse in the United States, 1992. Bethesda, MD: National Institutes of Health (NIH Publication Number 98-4327).

[57] Nestler, E..J. (2001) "Molecular Basis of Long-term Plasiticity Underlying Addiction", Nature Reviews Neuroscience, 2:119-28.

[58] O'Brien, C. (1976) "Experimental analysis of conditioning factors in human narcotic addiction," Pharmacological Review, 25:533-43. 
[59] O'Brien, C. (1997) “A Range of Research-Based Pharmacotherapies for Addiction," Science, 278:66-70.

[60] O'Donoghue, Ted, and Mathew Rabin (1999), “Doing It Now or Later," American Economic Review, (89):103-24.

[61] O'Donoghue, Ted, and Mathew Rabin (2000), "Addiction and Present-Biased Preferences," manuscript, Berkeley.

[62] Office of National Drug Control Policy (2001a). What American Users Spend on Illegal Drugs. Washington, DC: Executive Office of the President (Publication No. NCJ-192334).

[63] Office of National Drug Control Policy (2001b). The Economic Costs of Drug Abuse in the United States, 1992-1998. Washington, DC: Executive Office of the President (Publication No. NCJ-190636).

[64] Olds, J. and P. Milner (1954) "Positive reinforcement produced by electrical stimulation of septal area and other regions of rat brain," Journal of Comparative and Physiological Psychology, 419-27.

[65] Orphanides, Athanasios, and David Zervos (1995) "Rational Addiction with Learning and Regret," Journal of Political Economy, 103: 739-58.

[66] Panksepp, J. (1998) Affective Neuroscience: The Foundations of Human and Animal Emotions. New York: Oxford University Press.

[67] Pickens, R. and W.C. Harris (1968) "Self-administration of d-amphetamine by rats," Pscyhopharmacologia, 158-63.

[68] Robins, L. (1994) "Vietnam Veterans' Rapid Recovery from Heroin Addiction: a Fluke or Normal Expectation," Addiction, 1041-54.

[69] Robins, L., D. Davis, and D. Goodwin (1974) "Drug Use by U.S. Army Enlisted Men in Vietnam: a Follow-up on their Return Home," American Journal of Epidemiology, 235-49.

[70] Robinson. T. and K. Berridge (1993) "The Neural Basis of Drug Craving: An Incentive-Sensitization Theory of Addiction," Brain Research Reviews, 18(3):24791.

[71] Robinson, T. and K. Berridge (2000) "The psychology and neurobiology of addiction: an incentive sensitization view," Addiction, Suppl 2:91-117.

[72] Schultz, W. (1998) "Predictive reward signal of dopamine neurons," Journal of Neurophysiology, 80:1-27. 
[73] Schultz, Wolfram (2000) "Multiple Reward Signals in the Brain,"Nature Reviews Neurosicence, 1:199-207.

[74] Schultz, W., P. Dayan, and P.R. Montague (1997) "A neural substrate of prediction and reward," 275:1593-99.

[75] Trosclair, A., C. Huston, L. Pederson, and I. Dillon (2002), "Cigarette Smoking Among Adults - United States, 2000," Morbidity and Mortality Weekly Report, $51(29), 642-645$.

[76] Ungless, M., J. Whistler, R. Malenka, and A. Bonci (2001) "Single cocaine exposure in vivo induces long-term potentiation in dopamine neurons," Nature, 411(31):583-87.

[77] United States Census Bureau (2001). Statistical Abstract of the United States. Washington, DC: US Government Printing Office.

[78] Volkow, N.D. (1997) “The role of the dopamine system in addiction," Hospital Practice (Special reports), April: 22-26.

[79] Volkow, N.D., Wang, G.-J., Fowler, J.S. (1997) "Imaging studies of cocaine in the human brain and studies of the cocaine addict. In: Imaging Brain Structure and Function," in Annals of the New York Academy of Sciences, Lester D.S., Felfer C.C., Lewis E.N. (eds), 820:41-53.

[80] Vorel, S. and E. Garner (2001) "Drug Addiction and the Hyppocampus," Science, 294(9):1235a

[81] Vorel, S., et. al. (2001) "Relapse to Cocaine-Seeking After Hippcampal Theta Burst Stimulation," Science, 292:1175-78.

[82] Wegner, Daniel (1994) White Bears and other unwanted thoughts: Suppression, obsession, and the psychology of mental control. New York: Guildford Press.

[83] Wickelgren (1997) "Getting the Brain's Attention," Science, 278:35-37. 


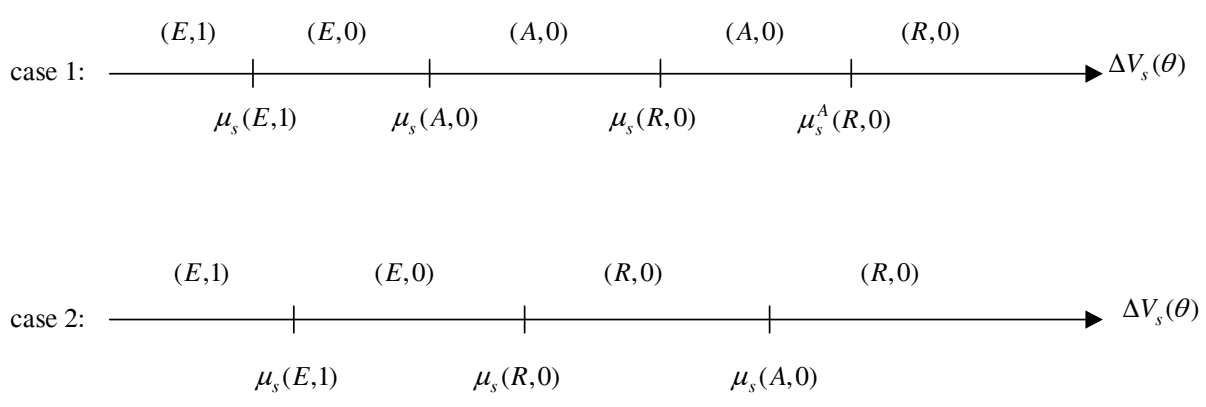

Figure 1: Optimal decision rule within a period. 

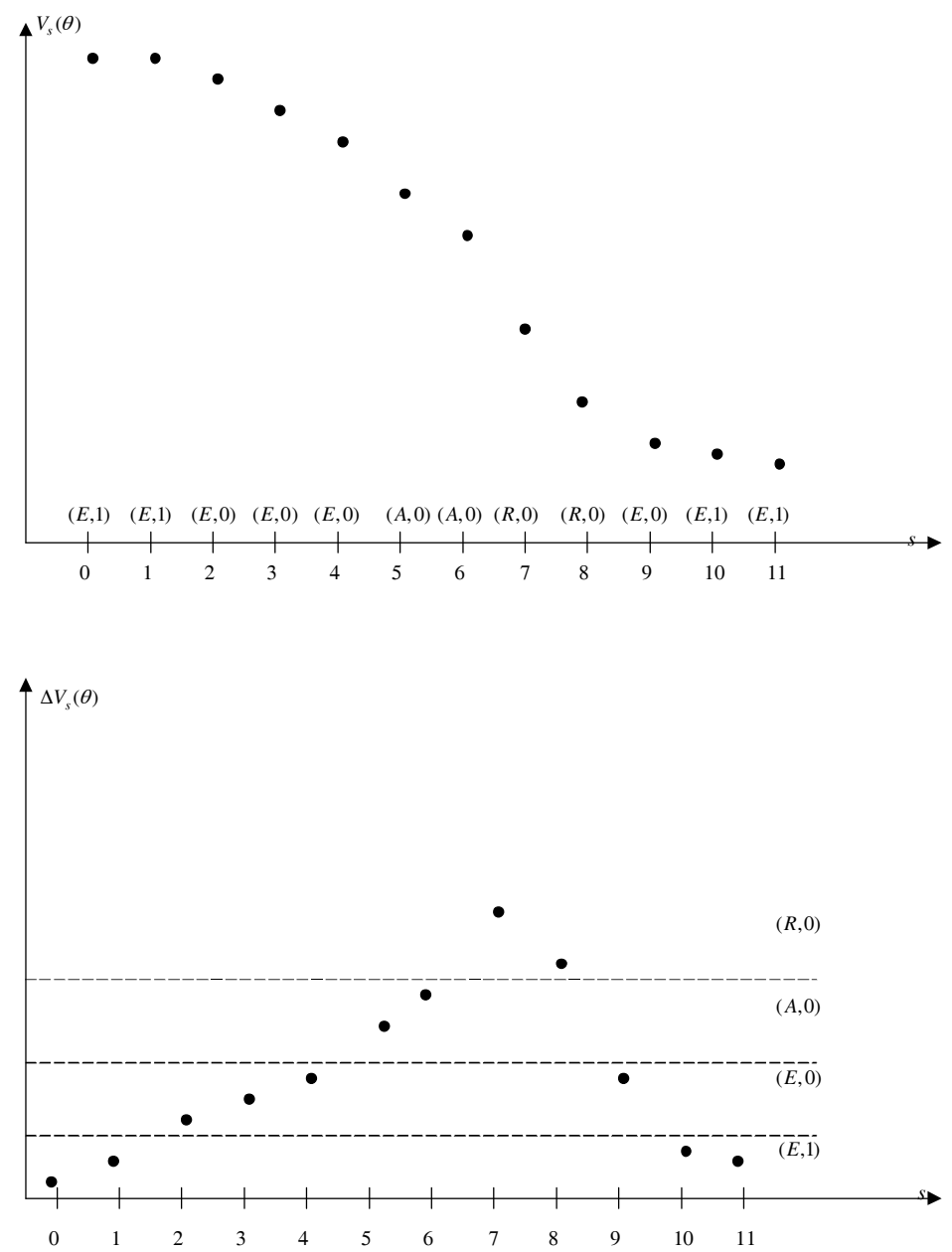

Figure 2: Graphical representation of the optimal decision rule. 